\title{
Methane dissociation on Pt(111): Searching for a specific reaction parameter density functional
}

Cite as: J. Chem. Phys. 144, 044702 (2016); https://doi.org/10.1063/1.4939520

Submitted: 23 October 2015 . Accepted: 20 December 2015 . Published Online: 22 January 2016

Francesco Nattino, Davide Migliorini, Matteo Bonfanti (D), and Geert-Jan Kroes (D)
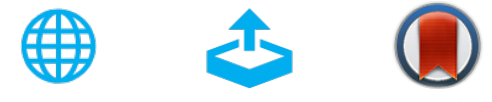

View Online

Export Citation

\section{ARTICLES YOU MAY BE INTERESTED IN}

Methane dissociation and adsorption on Ni(111), Pt(111), Ni(100), Pt(100), and Pt(110)-(1×2): Energetic study

The Journal of Chemical Physics 132, 054705 (2010); https://doi.org/10.1063/1.3297885

Methane dissociation on Ni(111) and Pt(111): Energetic and dynamical studies

The Journal of Chemical Physics 130, 054701 (2009); https://doi.org/10.1063/1.3065800

Mode-selective chemistry on metal surfaces: The dissociative chemisorption of $\mathrm{CH}_{4}$ on $\mathrm{Pt}(111)$

The Journal of Chemical Physics 144, 184709 (2016); https://doi.org/10.1063/1.4948941

The Journal 


\title{
Methane dissociation on $\mathrm{Pt}(111)$ : Searching for a specific reaction parameter density functional
}

\author{
Francesco Nattino, ${ }^{1, a)}$ Davide Migliorini, ${ }^{1}$ Matteo Bonfanti, ${ }^{2}$ and Geert-Jan Kroes ${ }^{1}$ \\ ${ }^{1}$ Leiden Institute of Chemistry, Leiden University, Gorlaeus Laboratories, P.O. Box 9502, \\ 2300 RA Leiden, The Netherlands \\ ${ }^{2}$ Dipartimento di Chimica, Università degli Studi di Milano, via Golgi 19, 20133 Milano, Italy
}

(Received 23 October 2015; accepted 20 December 2015; published online 22 January 2016)

\begin{abstract}
The theoretical description of methane dissociating on metal surfaces is a current frontier in the field of gas-surface dynamics. Dynamical models that aim at achieving a highly accurate description of this reaction rely on potential energy surfaces based on density functional theory calculations at the generalized gradient approximation. We focus here on the effect that the exchange-correlation functional has on the reactivity of methane on a metal surface, using $\mathrm{CHD}_{3}+\operatorname{Pt}(111)$ as a test case. We present new ab initio molecular dynamics calculations performed with various density functionals, looking also at functionals that account for the van der Waals (vdW) interaction. While searching for a semi-empirical specific reaction parameter density functional for this system, we find that the use of a weighted average of the PBE and the RPBE exchange functionals together with a vdW-corrected correlation functional leads to an improved agreement with quantum state-resolved experimental data for the sticking probability, compared to previous PBE calculations. With this semi-empirical density functional, we have also investigated the surface temperature dependence of the methane dissociation reaction and the influence of the rotational alignment on the reactivity, and compared our results with experiments. (C) 2016 AIP Publishing LLC. [http://dx.doi.org/10.1063/1.4939520]
\end{abstract}

\section{INTRODUCTION}

The level of accuracy that can be achieved in modeling gas-surface reaction dynamics strongly depends on the accuracy of the interaction potential employed. Assuming the Born-Oppenheimer approximation to be valid, the state-of-the-art electronic structure method that allows the computation of potential energy surfaces (PESs) for molecules interacting with metal surfaces is density functional theory (DFT) at the generalized gradient approximation (GGA) level. ${ }^{1}$ Many implementations of the GGA exist, differing in the approximate expression of the exchange-correlation functional $\left(E_{X C}\right)$. Even though functionals at the GGA level are not expected to be accurate in predicting, for instance, the reaction barrier height for a given system (for gas phase reactions the lowest mean absolute error is $3.8 \mathrm{kcal} / \mathrm{mol}$, as obtained with the MOHLYP2 functional ${ }^{2,3}$ ), semi-empirical versions of $E_{X C}$ allow one to produce accurate PESs through the fitting of one parameter to a single set of experimental data for $\mathrm{H}_{2}$-metal surface interactions. ${ }^{4-7}$

Methane dissociation on metal surfaces is a reaction for which an accurate theoretical description has not yet been achieved. ${ }^{7}$ Many of the studies that focused on this system $^{8-35}$ have been motivated by fundamental questions and focused on the hyperthermal energy ranges, but the reaction is also of industrial interest, as it is believed to represent a rate limiting step in the methane steam reforming process. ${ }^{36}$ Previous work ${ }^{27,37}$ suggested that the same direct reaction mechanism is followed under both thermal (catalytically

\footnotetext{
a)Email: f.nattino@chem.leidenuniv.nl
}

relevant) and hyperthermal conditions on $\mathrm{Ni}$ and $\mathrm{Pt}$, validating the use of a dynamical approach to study this reaction. Mode specificity ${ }^{13,30,35}$ with unusually large vibrational efficacies ${ }^{14,15}$ and bond selectivity for the partially deuterated isotopologues ${ }^{17,24,33}$ are just a few examples of the interesting properties of the sticking probabilities that have been observed for this system. Yoder et al. ${ }^{19,38,39}$ were also able to show that the reactivity of methane is influenced by its rotational alignment. They employed a linearly polarized laser to selectively prepare $\mathrm{CH}_{4}$ and $\mathrm{CHD}_{3}$ molecules in a particular rovibrational state and changed the laser polarization direction to generate different angular momentum (and vibrational transition dipole moment) alignments. Their results suggest that the reactivity depends mainly on the alignment of the vibrational transition dipole moment of the molecule, rather than on its angular momentum alignment, with the highest reactivity observed for the vibrational transition dipole moment oriented in the direction parallel to the surface.

We have previously shown ${ }^{40}$ that by using the PBE density functional $^{41,42}$ in combination with the $a b$ initio molecular dynamics (AIMD) method semi-quantitative agreement can be obtained with quantum state-resolved experimental sticking probabilities for $\mathrm{CHD}_{3}$ on $\mathrm{Pt}(111)$. In particular, we simulated two types of molecular beam experiments: molecular beams in which the molecules are initially vibrationally excited with one quantum in the $v_{1}$ ( $\mathrm{CH}$-stretch) mode through a laser and molecular beams of which the vibrational composition is determined by the gas temperature of the beam ("laseroff" experiments). Even though a large fraction of the molecules in the laser-off beams is in a vibrationally excited state (assuming no or minor vibrational cooling after the 
supersonic expansion), the vibrational state which is by far most populated is the vibrational ground state. For both laser-off and $\mathrm{CH}$-stretch excited conditions, we considered exclusively experiments conducted at relatively large average collision energies, so that the reaction occurs in the classical, over the barrier energy regime. ${ }^{40}$

The combination of the AIMD method employed and the experimental observables considered is suitable for testing the accuracy of the PES underlying the methane dissociation on $\mathrm{Pt}(111)$. In fact, the quasi-classical trajectory (QCT) technique can be used to accurately study the vibrational efficacy of the $\mathrm{CH}$-stretch mode, as this vibrational mode is off-resonance with other modes due to the mass mismatch between the $\mathrm{H}$ and the heavier D atoms. Therefore, less "artificial" intramolecular vibrational energy redistribution (IVR) caused by the use of classical mechanics is expected for this isotopologue of methane in the case of pre-excitation of the $\mathrm{CH}$-stretch mode. ${ }^{43,44}$ The use of the QCT technique is further justified because the average total energies (vibrational + translational) we consider here are larger than the zero-point energy (ZPE) corrected minimum energy barrier, below which the reaction could only occur through a non-physical energy flow of the vibrational ZPE to the reaction coordinate in classical mechanics. ${ }^{28}$ Furthermore, previous quantum calculations have suggested a minor influence of tunneling at large collision energies and a predominant over-the-barrier dissociation mechanism for the reaction of methane on a metal surface. ${ }^{21}$ Moreover, it has been shown ${ }^{31,32}$ that the strong isotope effect observed for methane reacting on $\operatorname{Pt}(111)^{9}$ could be explained purely on the basis of the different ZPEs that characterize the different isotopologues of methane. By using the AIMD method, in addition to avoiding fitting/interpolation inaccuracies in the pre-calculated potential due to the "on-the-fly" computation of the forces, no dynamical approximation with respect to the time evolution of any of the molecular degrees of freedom has to be made a priori ${ }^{40,45}$ Finally, the motion of the surface atoms and their thermal displacement from the equilibrium positions, which has been shown to play a role in the dissociation dynamics of methane on metal surfaces, ${ }^{30,40,46}$ can be explicitly included in the dynamics. In fact, the movement of the first layer surface atoms towards the impinging molecules significantly lowers the dissociation barrier for methane on metals, ${ }^{47-49}$ and this finding has been used to explain ${ }^{46,49}$ the extent to which the reactivity is enhanced by increasing the surface temperature $\left(T_{s}\right) .9,11,50-53$

The agreement with experimental data obtained using the PBE functional was limited: ${ }^{40}$ The reactivity computed when simulating laser-off molecular beams was overestimated over the whole energy range investigated, with the theoretical reaction probability curve being shifted from the experimental curve by about $0.1 \mathrm{eV}$. Furthermore, the vibrational efficacy obtained for the $\mathrm{CH}$-stretch mode of $\mathrm{CHD}_{3}$ was underestimated. These findings are not surprising given the limited accuracy of GGA-level density functionals, like PBE, as already mentioned above. In order to tackle these limitations, we present here a study of the influence of $E_{X C}$ on the dissociation of methane on $\operatorname{Pt}(111)$. Our ultimate goal is to develop a semi-empirical density functional for this system, following the specific reaction parameter (SRP) approach. This approach, inspired by the work of Truhlar and co-workers, ${ }^{54,55}$ has been successfully applied to the reactive scattering of $\mathrm{H}_{2}$ from low-index copper surfaces. ${ }^{4-6}$ To be considered as such, a SRP functional for the system we consider here must, in principle, produce good agreement with the laser-off experimental reaction probabilities over the energy range for which data are available, and it must correctly predict the reactivity of the $v_{1}=1$ vibrational state. Once such a semi-empirical density functional is identified, testing it against other experimental observables for the same system is helpful for ultimately validating its accuracy or ascertaining the validity of the dynamical approach used for describing these observables.

In the search of a SRP functional for this system, we have considered functionals that mimic the van der Waals (vdW) interaction, ${ }^{56}$ motivated by recent studies that demonstrated the improved description of two molecule-surface reactions achieved with vdW-corrected density functionals. ${ }^{57,58}$ Note that traditional GGA functionals are not able to predict the long range dispersion interactions. First, we have investigated the presence of the vdW physisorption well that appears if a vdW-corrected density functional is employed, and compared the well depth to experimental data to validate the vdW approach employed. Next, we have performed static calculations to identify the minimum reaction barrier height for each of the functionals considered. The most promising functionals have been tested through new AIMD calculations for the $\mathrm{CHD}_{3}+\mathrm{Pt}(111)$ system and the computed sticking probabilities have been compared to the previous PBE-AIMD calculations and to the results of quantum state-resolved experiments. ${ }^{40}$ Finally, the functional that returns the best agreement with the experimental sticking probabilities has been tested by investigating two additional observables. First, we have studied the $T_{s}$ dependence of the methane dissociation reaction and compared the computed results to experimental data from Luntz and Bethune. ${ }^{9}$ Second, we have looked at the influence of the rotational alignment on the reaction probability, simulating experiments similar to the one described in Refs. 19 and 38 but at larger collision energies, for which we can calculate statistically accurate reaction probabilities with AIMD.

The results indicate that the use of a density functional that employs the correlation functional of Dion et al. ${ }^{56}$ which is able to mimic vdW-type interactions, and a weighted average of the PBE and the RPBE ${ }^{59}$ exchange functionals, leads to improved agreement with experimental data when compared to the previous PBE-AIMD calculations. The improvements consist in (i) a decreased laser-off reactivity, (ii) a more gradual increase of the laser-off reactivity curve over the energy range for which experimental data are available, and (iii) an increased vibrational efficacy for the $\mathrm{CH}$ stretch mode of $\mathrm{CHD}_{3}$. The laser-off reaction probability curve obtained when using this functional, however, still increases somewhat too steeply with the collision energy so that the experimental reactivity is overestimated at the highest collision energies simulated. Interestingly, this new density functional returns a minimum barrier height that is almost equal to the PBE barrier height, indicating that aspects of the PES other than the barrier height also affect the reactivity. Our results 
suggest that the transition state region is less accessible to the reacting molecules as the dissociating bond at the transition state is more elongated than with the PBE functional. This also explains the larger and improved vibrational efficacy obtained for the $\mathrm{CH}$-stretch mode on the basis of one of Polanyi's rules, ${ }^{60}$ according to which the more extended the dissociating $\mathrm{CH}$ bond is at the transition state, the higher should be the vibrational efficacy. Regarding the comparison with other experimental observables, our dynamical model would seem to underestimate the extent to which the reactivity of $\mathrm{CH}_{4}$ on $\mathrm{Pt}(111)$ is enhanced by $T_{s}$, as measured by Luntz and Bethune. ${ }^{9}$ Furthermore, no rotational alignment effects have been obtained for $\mathrm{CHD}_{3}+\mathrm{Pt}(111)$ at the large collision energies investigated $\left(\left\langle E_{i}\right\rangle=0.83 \mathrm{eV}\right)$ for which, unfortunately, no experimental data are yet available.

The structure of this article is as follows. In Section II, the methodology is described. In Section III, all the results are presented and discussed: Static calculations that focus on the vdW well (Section III A) and on the dissociation barrier (Section III B), and AIMD calculations simulating quantum state-resolved experiments for $\mathrm{CHD}_{3}+\mathrm{Pt}(111)$ (Section III C) and investigating the $T_{S}$ dependence (Section III D) and the rotational alignment dependence (Section III E) of the methane dissociation reaction. Finally, the conclusions are presented in Section IV.

\section{METHODS}

The sticking (dissociation) probability $S_{0}$ of methane on $\operatorname{Pt}(111)$ has been determined using the AIMD technique. ${ }^{61,62}$ Details about the calculations have been given previously ${ }^{40}$ and are only briefly summarized here. A $(3 \times 3)$-surface-unitcell slab with 5 atomic layers is employed to model the surface, and a vacuum space $13 \AA$ long has been used to separate periodic replicas of the surface. The bulk lattice constant as well as the interlayer distances has been selfconsistently relaxed for each of the tested density functionals. The electronic structure calculations are characterized by an energy cutoff for the plane wave expansion equal to $350 \mathrm{eV}$. A $\Gamma$-centered $4 \times 4 \times 1 \mathrm{k}$-point grid has been employed to sample the first Brillouin zone, with the projector augmented wave (PAW) method ${ }^{63,64}$ to represent core electrons and a Fermi-Dirac smearing with width parameter $\sigma=0.1 \mathrm{eV}$. All the electronic structure and AIMD calculations have been performed with the Vienna ab initio simulation package (VASP). ${ }^{63,65-69}$ In order to mimic surface temperature effects, the optimized lattice constant has been expanded according to experimental information, ${ }^{70,71}$ in a way that is entirely analogous to the procedure used in Ref. 40. For all the tested functionals, the optimized lattice constant (reported in Table I) is in reasonable agreement with the experimental value of $3.916 \AA .^{70,71}$

Displacements and velocities are initially assigned to the surface atoms to simulate their thermal motion, with the exception of the atoms in the bottommost layer, which are kept fixed at their ideal positions during the dynamical calculations. The procedure employed to sample the surface initial conditions is essentially the same as described in
TABLE I. Optimized lattice constants (in $\AA$ ) for bulk Pt calculated using the various tested exchange-correlation functionals. The experimental value is from Refs. 70 and 71.

\begin{tabular}{lc}
\hline \hline$E_{X C}$ & $a$ \\
\hline PBE & 3.975 \\
RPBE & 3.991 \\
vdW-DF & 4.032 \\
optPBE-vdW & 3.990 \\
PBE-vdW & 4.014 \\
RPBE-vdW & 4.039 \\
RPBE:PBE/0.24:0.76 & 3.975 \\
RPBE:PBE/0.1:0.9-vdW & 4.017 \\
Experimental & 3.916 \\
\hline \hline
\end{tabular}

Refs. 40 and 62. Clean surface 1.5 ps long AIMD calculations in which the number of particles in the simulation box, the volume and the total energy are kept constant (NVE runs) are employed to equilibrate at least nine differently initialized surfaces at a specific $T_{s}$. For this first equilibration run, the initial displacements and velocities are assigned assuming the atoms to be independent harmonic oscillators, with the corresponding frequencies in the range $10 \mathrm{meV}<\omega_{\alpha j}<$ $16 \mathrm{meV}$ as determined from normal mode analysis for a single atom in an ideal slab (the indexes $\alpha$ and $j$ refer to the $x, y$, and $z$ degrees of freedom of a metal atom and to the atomic layer considered, respectively). Note that the $\omega_{\alpha j}$ values have been found to be quite insensitive to the density functional employed. The initial conditions for the surface atoms in the molecule-surface simulations are randomly selected from consecutive 1 ps long $N V E$ equilibration runs. The time step employed for the surface equilibration is $1 \mathrm{fs}$. Whenever the average $T_{s}$ calculated over all the equilibrated surfaces in the second equilibration runs did not agree within one standard deviation with the desired $T_{s}$, we have performed a velocity rescaling run followed by an additional $N V E$ equilibration run ( $0.8 \mathrm{ps}$ and $1 \mathrm{ps}$, respectively). A final $1 \mathrm{ps}$ long equilibration run is then performed and employed to sample the surface initial conditions for the molecule-surface simulations. At the two highest $T_{s}$ 's simulated ( $850 \mathrm{~K}$ and $1200 \mathrm{~K}$ ), we have observed stacking fault defects occurring during some of the equilibration runs, and we have taken care of discarding such runs.

Between 500 and 2000 NVE trajectories have been computed to estimate $S_{0}$ for each molecular beam experiment simulated. The initial conditions of the trajectories are chosen such that the velocity of the center of mass of the molecule samples a flux-weighted velocity distribution that accounts for the spread in velocity of the beam. ${ }^{72}$ The parameters defining the velocity distributions (the stream velocity $v_{s}$ and the width parameter $\alpha^{72}$ ) of the quantum state-resolved experiments from Beck and co-workers have been determined by the experimentalists using time-of-flights (TOFs) techniques (all beam parameters have been reported and discussed in Ref. 40).

With respect to our previous work, we have slightly modified the operational definition of a reactive event. A trajectory is considered reacted if a $\mathrm{CH}$ or $\mathrm{CD}$ bond becomes larger than $2 \AA$ and remains in this configuration for at least 
$100 \mathrm{fs}$, or, alternatively, if a $\mathrm{CH}$ or $\mathrm{CD}$ bond becomes larger than $3 \AA$. This operational definition accounts, at least in part, for the possibility of barrier recrossing. No considerable difference has been found if this definition of reactive event is applied to the previous PBE-AIMD calculations. A trajectory is considered scattered if the center of mass of the molecule reaches the distance of $6 \AA$ from the surface, with the velocity pointing away from the surface. Trajectories are time propagated until one of the two mentioned outcomes is achieved. In very few trajectories of all the AIMD trajectories computed using vdW-corrected density functionals (6 out of more than 15000), the molecule remains trapped in the physisorption well after having collided with the surface. After a maximum propagation time of $1 \mathrm{ps}$, these molecules are considered scattered, on the basis of the negligible reactivity of physisorbed molecules on $\operatorname{Pt}(111)$ observed experimentally. ${ }^{73}$ Error bars on the sticking probability values have been estimated using the Wilson method ${ }^{74}$ and represent $95 \%$ confidence interval unless otherwise stated. The size of the error bars illustrates the quality of the statistical sampling for each sticking probability value computed, the error bars being a function of the estimated sticking probability itself and of the number of trajectories employed to estimate it.

In addressing the vibrational population of the supersonic molecular beams simulated, two types of beams have been considered. In the first beam type, the vibrational ground state is the most populated state, but vibrationally excited molecules are also present due to collisions with a heated nozzle ("laser-off" beams). In order to simulate these beams, we have assumed the vibrational population to be Boltzmannlike with a vibrational temperature equal to the vibrational temperature characterizing the beam. In the second class of beam, the $\mathrm{CHD}_{3}$ molecules are selectively prepared with one quantum of vibration in the $\mathrm{CH}$-stretch $\left(v_{1}\right)$ mode using a laser. Only molecules in the $v_{1}=1$ vibrational state have been used to simulate this second type of beam. Standard Monte Carlo techniques are employed to represent the initial vibrational state of the molecule, as already described in Ref. 40. We exploit the QCT method, which means that vibrational ZPE is initially imparted to the impinging molecules. The angular momentum of the molecule is initially set to zero with exclusion of the simulations of rotationally aligned beams, for which we have simulated $\mathrm{CHD}_{3}$ molecules in a particular $(J, K, M)$ state. The three quantum numbers reflect the quantization of the magnitude of the angular momentum $|\mathbf{J}|$, the projection of $\mathbf{J}$ on the molecule's figure axis $J_{\text {axis }}$, and the projection of $\mathbf{J}$ on the space fixed $z$-axis, $J_{z}$, respectively. In order to simulate a molecule in a particular $(J, K, M)$ state, first we have set the angular momentum of the molecule in the figure axis reference frame such that $|\mathbf{J}|=\hbar \sqrt{J(J+1)}$ and that $J_{\text {axis }}=\hbar K .^{75,76}$ We have next rotated the figure axis reference frame such that $J_{z}=\hbar M$ (the $z$ axis has been chosen to coincide with the surface normal). Therefore, the constraints on $|\mathbf{J}|, J_{\text {axis }}$, and $J_{z}$ determine three out of the six degrees of freedom needed to define $\mathbf{J}$ and the molecule's orientation: the magnitude of $\mathbf{J}$, the polar angle that defines the orientation of $\mathbf{J}$ with respect to the $z$ axis (equal to $\arccos \left(\frac{M}{\sqrt{J(J+1)}}\right)$ ), and one of the Euler angles defining the molecule's orientation with respect to the angular momentum (equal to $\arccos \left(\frac{K}{\sqrt{J(J+1)}}\right)$ ). The three remaining degrees of freedom, i.e., the azimuthal angle that defines the orientation of $\mathbf{J}$ with respect to the $z$ axis and the two remaining Euler angles that describe the molecule's orientation relative to $\mathbf{J}$ randomly sample the range $[0 ; 2 \pi]$.

Two types of functionals have been employed to represent the exchange-correlation functional in the electronic structure calculations. The first type of functional employed is characterized by PBE correlation. ${ }^{41,42}$ Apart from the PBE functional itself, we have considered the RPBE functional ${ }^{59}$ and the use of a density functional that employs PBE correlation and an exchange functional of the following form:

$$
E_{X}=x \cdot E_{X}^{R P B E}+(1-x) \cdot E_{X}^{P B E},
$$

where $E_{X}^{P B E}$ and $E_{X}^{R P B E}$ are the PBE and the RPBE exchange functionals, respectively. The RPBE functional is known to be more repulsive than the PBE functional. By using a functional as in Equation (1), the barrier height of a system can be tuned through a change in the value of the $x$ coefficient. ${ }^{4,5}$

Wijzenbroek and Kroes ${ }^{57}$ have recently shown that vdWcorrected density functionals can improve the description of a molecule-surface reaction $\left(\mathrm{H}_{2}+\mathrm{Ru}(0001)\right)$. Moreover, $\mathrm{vdW}$-corrected density functionals have been shown to better describe properties such as adsorption energy and reaction barriers for $\mathrm{N}_{2}$ interacting with $\mathrm{W}(110)$, compared to traditional density functionals like PBE and RPBE. ${ }^{58}$ Motivated by these findings, the second class of functionals that we have tested is characterized by the non-local correlation functional proposed by Dion et al. ${ }^{56}$ to mimic dispersion interactions (van der Waals forces), which are generally not correctly described with regular GGA functionals. Note that an efficient implementation of this non-local correlation functional has recently become available. ${ }^{69,77}$ This correlation functional (from now on called vdW correlation) has originally been paired with revPBE ${ }^{78}$ exchange, forming the so-called vdWDF exchange-correlation functional, ${ }^{56}$ but it can, in principle, be used in combination with any exchange functional. ${ }^{79,80}$ In the current work, in addition to the vdW-DF functional, we have tested vdW correlation paired with PBE exchange (PBE$v d W$ ), with RPBE exchange (RPBE-vdW), with optPBE exchange (optPBE-vdW), ${ }^{80}$ and with an exchange functional of the form described by Equation (1). Note that the optPBE exchange functional is also defined as a weighted average of the PBE and the RPBE exchange expressions, with an additional modification of the $\kappa$ and $\mu$ parameters which appear in both the PBE and the RPBE exchange functionals. ${ }^{80}$

Barrier heights have been determined with the climbing image nudged elastic band (CINEB) method, ${ }^{81,82}$ as implemented in the VASP transition state tools developed by Henkelman and Jónsson. Nave and Jackson explored different reaction paths for methane dissociating on $\operatorname{Pt}(111) .{ }^{48,49}$ They identified the reaction pathway that leads to the adsorption of both the methyl group and the hydrogen atom on top sites as the path with the lowest energy transition state (TS). We have considered the same path for the CINEB calculations, optimizing four images between a methane molecule placed with its center of mass $4 \AA$ far from the surface (the reactant 
state) and the dissociated configuration (the product state). The path has been optimized until the forces acting on all the images became smaller than $15 \mathrm{meV} / \AA$. For the PBE functional, the use of a smaller threshold for convergence ( $5 \mathrm{meV} / \AA$ ) has been found to affect the transition state energy by less than $2 \mathrm{meV}$. Note that for all the tested functionals the slab employed has been constructed using the optimized bulk lattice constant (and optimized interlayer distances). Note also that the surface atoms have been kept fixed at their equilibrium positions during the CINEB calculations.

\section{RESULTS AND DISCUSSION}

\section{A. The van der Waals well}

As mentioned earlier, regular GGA density functionals are known to fail at describing dispersion interactions. As a consequence, functionals like PBE or RPBE do not correctly model the physisorption of molecules on metal surfaces. Figure 1(a) shows the interaction energy calculated as a function of the distance between the center of mass of $\mathrm{CH}_{4}$ and an ideal $\operatorname{Pt}(111)$ slab ( $Z$ coordinate). The geometry of the molecule has been kept frozen at the optimized gas-phase configuration. The molecule is placed above the hcp hollow site with one hydrogen atom pointing away from the surface $\left(\theta=0^{\circ}\right)$ and the other $\mathrm{H}$ atoms oriented toward the top sites. Note that the zero of energy is defined as the energy of a methane molecule in its gas-phase relaxed geometry placed midway between two periodic replicas of the slab $(Z=6.5 \AA)$. Using the PBE functional, only a shallow potential well that is about 20 meV deep is observed at $Z \approx 4 \AA$ (Figure 1(a)). Using the vdW correlation functional that accounts for dispersion interactions, a considerably deeper adsorption well is obtained (Figure 1(a)). As expected from the more repulsive nature of the RPBE exchange with respect to the PBE exchange, the vdW well calculated with the PBE-vdW functional is deeper and the minimum is slightly closer to the surface than for the RPBE-vdW functional (Figure 1(a)). The energy profile for large $Z$, however, is very similar for the two vdW-corrected functionals: at $Z=4.5 \AA$ the interaction energy calculated with the two functionals differs by only $10 \mathrm{meV}$, and this difference becomes even smaller at larger values of $Z$. This is consistent with the fact that the correlation term in $E_{X C}$ (which is the same for PBE-vdW and RPBE-vdW) and not the exchange term is responsible for the interaction at the largest distances. 56

In Figure 1(c), we have plotted two 1D cuts of the PES calculated for the same molecular configuration chosen for Figure 1(a), using the PBE-vdW functional but employing different amounts of vacuum space between the slab and its periodic replica. The curve calculated with the setup characterized by the smaller vacuum space $(13 \AA)$ is shifted upwards by about $45 \mathrm{meV}$ with respect to the other curve. For each computational setup, the zero of the interaction energy is defined as the energy of a methane molecule in its gas-phase configuration placed midway between two periodic replicas of the slab. Note that for the system with $30 \AA$ of vacuum space, at $Z=6.0 \AA$, the interaction energy is still about $45 \mathrm{meV}$. Therefore, the shift between the two curves
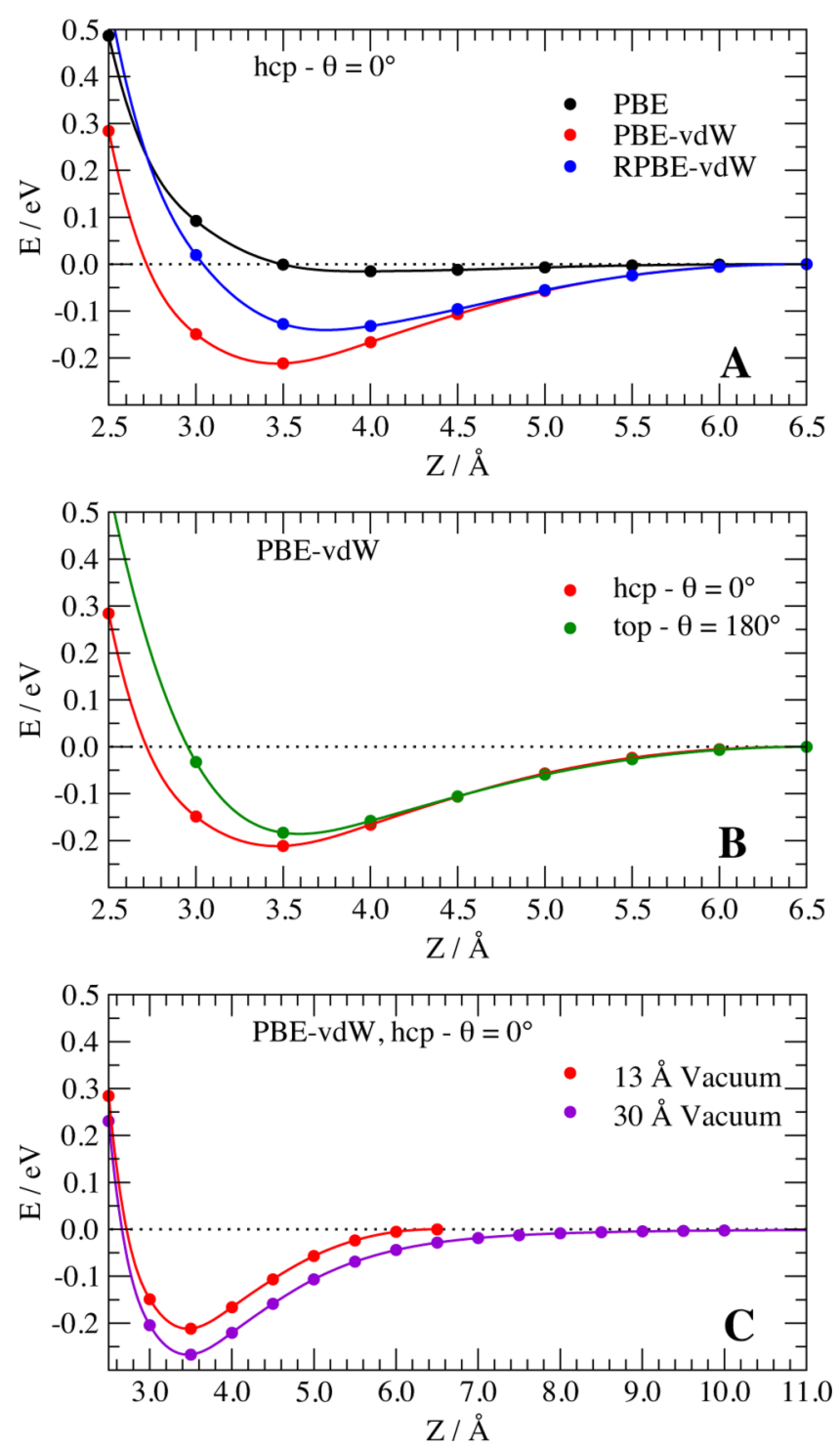

FIG. 1. Interaction energy as a function of the distance of $\mathrm{CH}_{4}$ from an ideal $\mathrm{Pt}(111)$ surface. We compare the energy profiles for three density functionals (a), for two molecular orientations and surface impact sites (b), and for two computational setups differing only in the amount of vacuum space between two periodic replicas of the slab (c).

at $Z=6 \AA$ is due to a residual attractive interaction for the gas-phase configuration of the setup with the smaller vacuum space. Unfortunately, the use of a larger vacuum space leads to an increase in the computational cost of the electronic structure calculations caused by the increased plane wave basis set size. Moreover, performing dynamics with the molecule initially placed at larger distances from the surface requires more time propagation steps for the molecule to reach the surface (and for the molecule to reach again the gas-phase after the impact with the surface, in the case of a scattering event). We have estimated that adding $3 \AA$ of vacuum space to our computational setup would overall increase the computational cost of a scattered trajectory by about $50 \%$. On the other hand, using $13 \AA$ of vacuum space affects the energetic by only a systematic $45 \mathrm{meV}$ upward shift of the gas-phase level, while it allows to keep the computational cost relatively low. We only have to keep in mind that the presented values of 
adsorption energies and energy barriers calculated using the $\mathrm{vdW}$ correlation functional apply to the computational setup that we have employed, while with the use of a larger vacuum space the adsorption energies are expected to be about $45 \mathrm{meV}$ larger and the barriers $45 \mathrm{meV}$ lower.

In Figure 1(b), we plot similar energy profiles as shown in Figure 1(a) but for two different combinations of adsorption site and molecular orientation. The functional employed is PBE-vdW. The two curves are only slightly different, and the biggest differences are observed at closer distances from the surface, suggesting that the multidimensional PES calculated using the vdW correlation functional is rather independent of $X$ and $Y$, and of the Euler angles of orientation in the proximity of the $\mathrm{vdW}$ (physisorption) well. We have optimized the molecular geometry testing various surface impact sites and molecular orientations and we have found that the configuration that corresponds to the minimum of the curves in Figure 1(a) is the lowest in energy $\left(E_{a d s}=0.214\right)$ while the adsorption energy differs by less than $43 \mathrm{meV}(1 \mathrm{kcal} / \mathrm{mol})$ if other molecular adsorption configurations (including other local minima) are considered. Note that for all the adsorption configurations considered the geometry of the molecule does not considerably differ from the gas-phase configuration. In Table II, we report the molecular adsorption energy $E_{a d s}$ calculated with three different exchange correlation functionals: PBE-vdW, RPBE$\mathrm{vdW}$, and a functional that employs the $\mathrm{vdW}$ correlation and the exchange of the form of Equation (1) with $x=0.1$ (the choice of this value for the $x$ coefficient will become clearer later on). Consistently with the curves reported in Figure 1(a), the adsorption energy calculated with the PBE-vdW functional is about $60 \mathrm{meV}$ larger than the RPBE-vdW value, while the RPBE:PBE/0.1:0.9-vdW functional returns an intermediate value. As mentioned earlier in this section, these values of $E_{a d s}$ are expected to be about $45 \mathrm{meV}$ larger for a computational setup with a larger amount of vacuum space.

Experimentally, methane is known to molecularly adsorb on $\mathrm{Pt}(111)$ with an adsorption energy in the range $0.17 \mathrm{eV}-0.23 \mathrm{eV}^{83-87}$ (see also Table II). The values of $E_{A d s}$ reported in Table II are in fairly good agreement with this experimental range. Considering the extra $45 \mathrm{meV}$ that would have to be added when considering a larger vacuum space, the PBE-vdW and the RPBE:PBE/0.1:0.9-vdW functionals would slightly overestimate the adsorption energy, whereas the adsorption energy calculated with the RPBE-vdW functional would fall inside the experimental range. The reasonable agreement obtained between the vdW approach employed and the experimental data in the physisorption well depth

TABLE II. Adsorption energies (in eV) corresponding to the bottom of the van der Waals well calculated with various density functionals. The experimental range includes all the values from Refs. 83-87.

\begin{tabular}{ll}
\hline \hline$E_{X C}$ & $E_{\text {Ads }}$ \\
\hline PBE-vdW & 0.214 \\
RPBE-vdW & 0.141 \\
RPBE:PBE/0.1:0.9-vdW & 0.205 \\
Experimental range & $0.17-0.23$ \\
\hline \hline
\end{tabular}

suggests that the description of the long-range interaction achieved with the method of Dion et al. ${ }^{56}$ for this system is physically sound.

We note in passing that accurately modeling the vdW well for methane interacting with metal surfaces should be relevant for describing two recent sets of experimental data. ${ }^{73,88}$ In a first study, Beck and co-workers investigated the physisorption of $\mathrm{CH}_{4}$ and the dissociation of $\mathrm{CH}_{4}$ physisorbed on $\mathrm{Pt}(111)$, without finding significant differences between laser-off and vibrationally excited beams. ${ }^{73}$ In a second study, Utz and coworkers suggested a precursor-mediated dissociation channel for $\mathrm{CH}_{4}$ impinging at very low collision energies on $\operatorname{Ir}(111) .{ }^{88}$ To our knowledge all dynamical studies that have appeared so far relied on density functionals that fail at modeling dispersion interactions, and therefore cannot be used to model these two sets of experimental data.

\section{B. The dissociation barrier}

Using CINEB calculations, we have identified the TS along a reaction path that connects a molecule in its equilibrium geometry placed at $Z=4 \AA$ to a dissociated configuration corresponding to a methyl fragment and a hydrogen atom adsorbed on two neighboring top sites, with one $\mathrm{CH}$ bond of the methyl group pointing toward the adsorbed $\mathrm{H}$. This path, illustrated in Figure 2, has been suggested by Nave et al. to correspond to the minimum energy path for dissociation of $\mathrm{CH}_{4}$ on $\mathrm{Pt}(111),{ }^{49}$ as also predicted by Lozano et al. $^{32}$ In both Refs. 49 and 32, six reaction paths differing in the orientation of the dissociating $\mathrm{CH}$ bond (towards a bridge, a hcp-hollow, or a fcc-hollow site) and of the other (non-reacting) $\mathrm{CH}$ bonds have been considered. Very similar TSs have been found across the different paths, with all the TS energies falling within $50 \mathrm{meV}$ from the minimum energy barrier in both studies. ${ }^{32,49}$ For this reason, we have focused here only on the path characterized by the lowest energy TS, as we expect that the other TSs would be affected in a similar way by the choice of the density functional.

In Figure 2, we plot the energy computed along the dissociation path for the various tested functionals. Note that the density functionals that account for the $\mathrm{vdW}$ interaction predict negative energies on the reactant side of the barrier, as expected from the presence of a vdW well (see Section III A). Product energies can also vary significantly, and the dissociation path considered is predicted to be endothermic or slightly exothermic depending on the functional employed.

Some details about the TSs obtained are summarized in Tables III and IV, where we report the TS energy (with and without zero-point energy corrections) and some descriptors of the TS geometry: the distance of the $\mathrm{C}$ atom to the surface $Z_{C}$, the distance of the dissociating $\mathrm{H}$ atom to the surface $Z_{H}$, the angle included between the dissociating $\mathrm{CH}$ bond and the surface normal $\theta_{\text {diss }}$, the angle $\beta$ that the "umbrella" axis (i.e., the axis of the non-dissociating $\mathrm{CH}_{3}$ moiety) forms with the surface normal, and the length of the dissociating $\mathrm{CH}$ bond, $r_{b}$. Note again that the TS energies calculated using the $\mathrm{vdW}$ correlation functional are expected to be about $45 \mathrm{meV}$ lower if a large enough vacuum space is included between periodic replicas of the slab (see Section III A). 

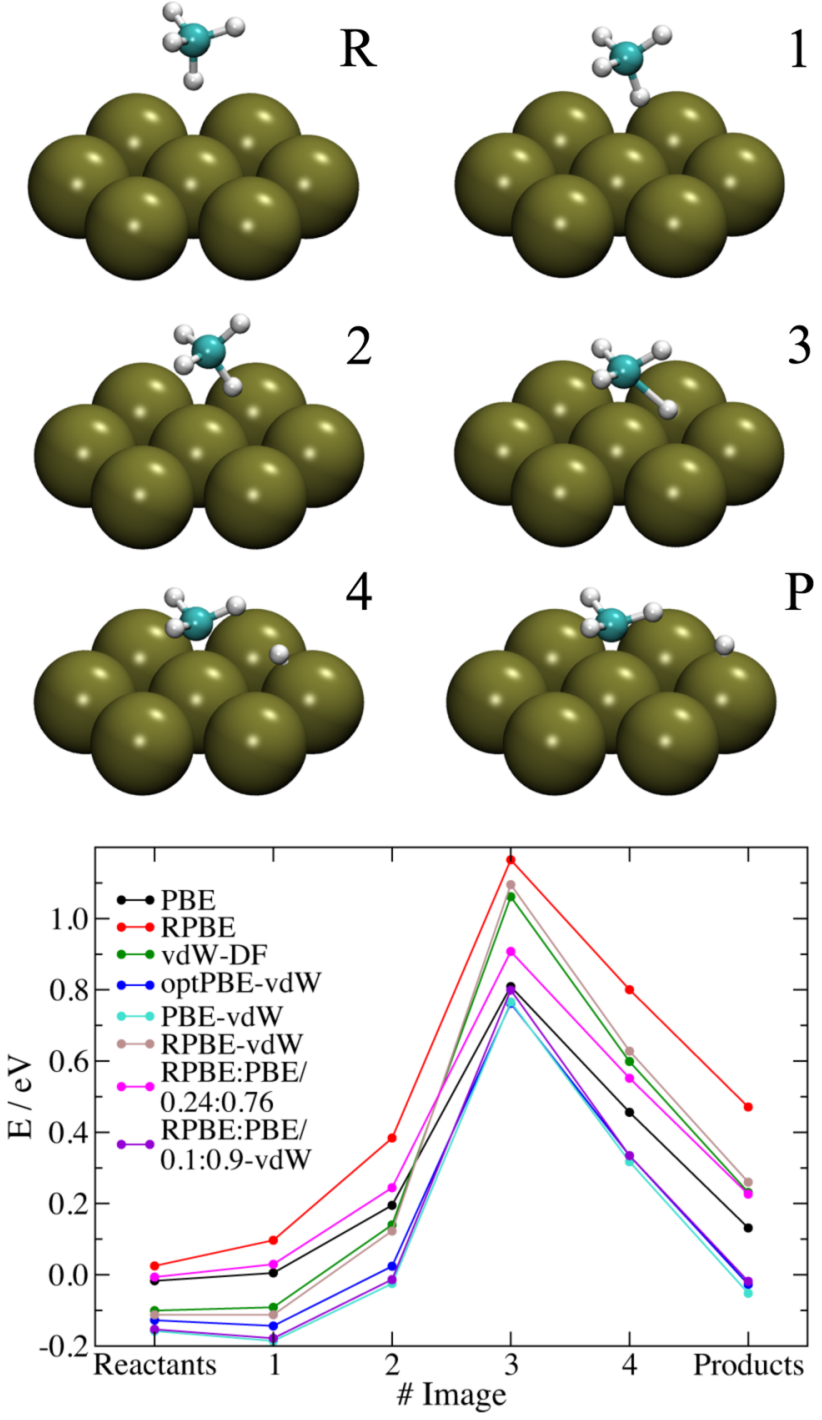

FIG. 2. Above: Illustration of the molecular configurations that correspond to the images resulting from CINEB calculations. Below: The energy of each image is plotted as a function of the image number ( $R$ and $P$ are for reactants and products, respectively). The colors distinguish the various density functionals (lines are for guiding the eye).

We observe that the geometries of the TSs identified do not differ considerably in the values of $\theta_{\text {diss }}, \beta$, and $Z_{H}$, while $Z_{C}$ and $r_{b}$ are the most affected by the choice of the density functional employed. We also observe that ZPE corrections affect the barrier heights for the $\mathrm{CH}_{4}$ dissociation with a shift downwards of about $0.12 \mathrm{eV}$, which is almost independent of the density functional employed. In the case of a different isotopologue of methane, like $\mathrm{CHD}_{3}$, different $\mathrm{ZPE}$ corrections apply, which also depend on whether a $\mathrm{CH}$ bond or a $\mathrm{CD}$ bond is dissociating, and, for the case of CD bond dissociation, on the position of the $\mathrm{H}$ atom (see Table III). For $\mathrm{CHD}_{3}$, the ZPE-corrected barrier for $\mathrm{CH}$ bond dissociation is lower than the barrier for $\mathrm{CD}$ bond dissociation, which is consistent with the larger ZPE associated with the $\mathrm{CH}$ bond in the gas-phase, as already observed previously. ${ }^{25,31,35,40,89}$ The two possible configurations for $\mathrm{CD}$ bond cleavage (symmetric or non-symmetric with respect to the plane perpendicular to the surface including the $\mathrm{C}$ atom and the dissociating $\mathrm{D}$ atom)
TABLE III. Transition state energies calculated with various density functionals for methane reacting on $\operatorname{Pt}(111) . E_{b}^{c}$ and $E_{b}$ are the minimum barrier heights with and without zero-point energy corrections (in eV). For $\mathrm{CHD}_{3}$, we consider the configuration with the dissociating $\mathrm{CH}$ bond $\left(\mathrm{CD}_{3}-\mathrm{H}\right)$ and the two configurations with the dissociating $\mathrm{CD}$ bond $\left(\mathrm{CHD}_{2}-\mathrm{D}\right)$, symmetric and non-symmetric with respect to the plane perpendicular to the surface containing the $\mathrm{C}$ atom and the dissociating $\mathrm{D}$ atom.

\begin{tabular}{lccccc}
\hline \hline & & \multicolumn{5}{c}{$E_{b}^{c}$} \\
\cline { 3 - 6 } & & & & $\mathrm{CHD}_{2}-\mathrm{D}$ & $\mathrm{CHD}_{2}-\mathrm{D}$ \\
$E_{X C}$ & $E_{b}$ & $\mathrm{CH}_{4}$ & $\mathrm{CD}_{3}-\mathrm{H}$ & $(\mathrm{Sym})$ & $($ Asym) \\
\hline PBE & 0.809 & 0.687 & 0.682 & 0.729 & 0.729 \\
RPBE & 1.165 & 1.042 & 1.038 & 1.084 & 1.085 \\
vdW-DF & 1.061 & 0.942 & 0.936 & 0.983 & 0.983 \\
optPBE-vdW & 0.762 & 0.638 & 0.633 & 0.680 & 0.680 \\
PBE-vdW & 0.766 & 0.643 & 0.639 & 0.685 & 0.686 \\
RPBE-vdW & 1.096 & 0.976 & 0.970 & 1.017 & 1.017 \\
RPBE:PBE/0.24:0.76 & 0.908 & 0.785 & 0.780 & 0.827 & 0.828 \\
RPBE:PBE/0.1:0.9-vdW & 0.799 & 0.679 & 0.674 & 0.720 & 0.721 \\
\hline \hline
\end{tabular}

have almost identical ZPE-corrected dissociation barriers. ${ }^{89}$ In general, the ZPE corrections for the same isotopologue, dissociating bond, and molecular configuration are almost independent of the functional employed. Therefore, the nonZPE-corrected barrier $E_{b}$ can be considered when comparing the various functionals for a particular isotopologue and dissociating bond.

The TS geometry that we have found using the PBE functional is very similar in the values of $Z_{C}, Z_{H}, \theta_{d i s s}$, and $r_{b}$ to the barrier geometry found by Nave et al. ${ }^{49}$ Small differences could be expected from the different computational setups (surface unit cell size, number of atomic layers, cutoff energy, k-point grid). The TS energy that we have found, however, is about $0.12 \mathrm{eV}$ lower than the value of Ref. 49. This difference can be explained accounting for the different surface unit cell sizes and the different number of atomic layers, as already discussed previously. ${ }^{40,45}$ Our computational setup is very similar to the one employed by Lozano et al., ${ }^{32}$ and the TS that we have found using the PBE functional is very similar to the minimum energy TS that they have obtained in both

TABLE IV. Transition state properties calculated with various density functionals for methane reacting on $\operatorname{Pt}(111) . Z_{C}$ and $Z_{H}$ are the distance to the surface of the $\mathrm{C}$ atom and of the dissociating $\mathrm{H}$ atom (in $\AA$ ), respectively. Furthermore, $\theta_{\text {diss }}$ and $\beta$ are the angles that the dissociating $\mathrm{CH}$ bond and the axis of the non-dissociating $\mathrm{CH}_{3}$ moiety form with the surface normal (in degrees), respectively, and $r_{b}$ is the length of the dissociating $\mathrm{CH}$ bond at the transition state (in $\AA$ ).

\begin{tabular}{lccccc}
\hline \hline$E_{X C}$ & $Z_{\boldsymbol{C}}$ & $Z_{\boldsymbol{H}}$ & $\theta_{\text {diss }}$ & $\beta$ & $r_{b}$ \\
\hline PBE & 2.249 & 1.222 & 133.2 & 170.5 & 1.502 \\
RPBE & 2.274 & 1.218 & 133.5 & 169.9 & 1.534 \\
vdW-DF & 2.307 & 1.220 & 134.0 & 168.5 & 1.566 \\
optPBE-vdW & 2.278 & 1.224 & 133.5 & 169.3 & 1.531 \\
PBE-vdW & 2.286 & 1.217 & 133.9 & 169.2 & 1.541 \\
RPBE-vdW & 2.313 & 1.219 & 134.1 & 168.4 & 1.572 \\
RPBE:PBE/0.24:0.76 & 2.257 & 1.222 & 133.2 & 170.3 & 1.512 \\
RPBE:PBE/0.1:0.9-vdW & 2.288 & 1.225 & 133.3 & 168.3 & 1.550 \\
\hline \hline
\end{tabular}


energy (0.81 eV vs. $0.809 \mathrm{eV})$ and geometry (e.g., $r_{b}=1.51 \AA$ vs. $r_{b}=1.502 \AA, \theta_{\text {diss }}=131^{\circ}$ vs. $\theta_{\text {diss }}=133.2^{\circ}$ ).

The overestimation of the laser-off reactivity using the PBE functional made us suggest that the PBE functional underestimates the dissociation barrier by about $0.1 \mathrm{eV}^{40} \mathrm{~A}$ popular density functional that is known to produce higher reaction barriers is the RPBE functional. Viñes et al. ${ }^{90}$ reported a TS energy of $1.06 \mathrm{eV}$ for the RPBE functional and a computational setup similar to the one that we have used. Here, we have also obtained a larger $E_{b}$ value for the RPBE functional (about $1.17 \mathrm{eV}$, see Table III). The discrepancy could be explained if the barrier reported in Ref. 90 would include surface relaxation effects, which is not explicitly mentioned. In fact, the upward motion of the surface atom below the dissociating molecule has been shown to be able to lower the barrier by up to $0.15 \mathrm{eV}$ for this system. ${ }^{49}$

The barrier that we have computed using the RPBE functional is about $0.36 \mathrm{eV}$ higher than the PBE barrier, as expected from the more repulsive nature of the exchange part of the former functional. Considering the $0.1 \mathrm{eV}$ energy shift between the experimental reaction probability curve and the PBE simulations, we expect the RPBE functional to highly overestimate the barrier height for this system. We note in passing that the RPBE barrier is slightly "later" than the PBE barrier, with $r_{b}$ being about $35 \mathrm{m \AA}$ more elongated. Calculations employing PBE correlation together with an exchange functional of the form of Equation (1) with a mixing coefficient $x=0.24$ have produced a barrier of $0.91 \mathrm{eV}$ (see Table III), approximately $0.1 \mathrm{eV}$ higher than the $E_{b}$ value obtained with the PBE functional.

Tables III and IV also include the results of CINEB calculations performed with various density functionals that employ vdW correlation. The originally proposed vdW-DF density functional, which exploits revPBE ${ }^{78}$ exchange, returns a TS energy which is similar to the one obtained using the RPBE-vdW functional (35 meV difference). Similarly, the optPBE-vdW and the PBE-vdW functionals produce almost identical barrier heights (4 meV difference). The TS energy calculated using the PBE-vdW (RPBE-vdW) functional is lower than the corresponding PBE (RPBE) value. Interestingly, dynamics performed using the PBE-vdW functional returned a laser-off reactivity slightly lower than the PBE one, but still overestimating the experimental values (see below). By mixing PBE exchange with more repulsive RPBE exchange (mixing coefficient $x=0.1$ ), we obtain a barrier which is about $35 \mathrm{meV}$ higher than the PBE-vdW value. Note that the TS states obtained using the PBE-vdW and the RPBE-vdW functionals are characterized by somewhat larger $r_{b}$ values than the PBE and the RPBE transition states, respectively.

\section{The simulation of quantum-state resolved experiments}

We have previously shown ${ }^{40}$ that the sticking probability $S_{0}$ calculated for $\mathrm{CHD}_{3}$ on $\mathrm{Pt}(111)$ using AIMD in combination with the PBE functional gave semi-quantitative agreement with experimental data (see also Figure 3(a)). The discrepancies between theory and experiment can be
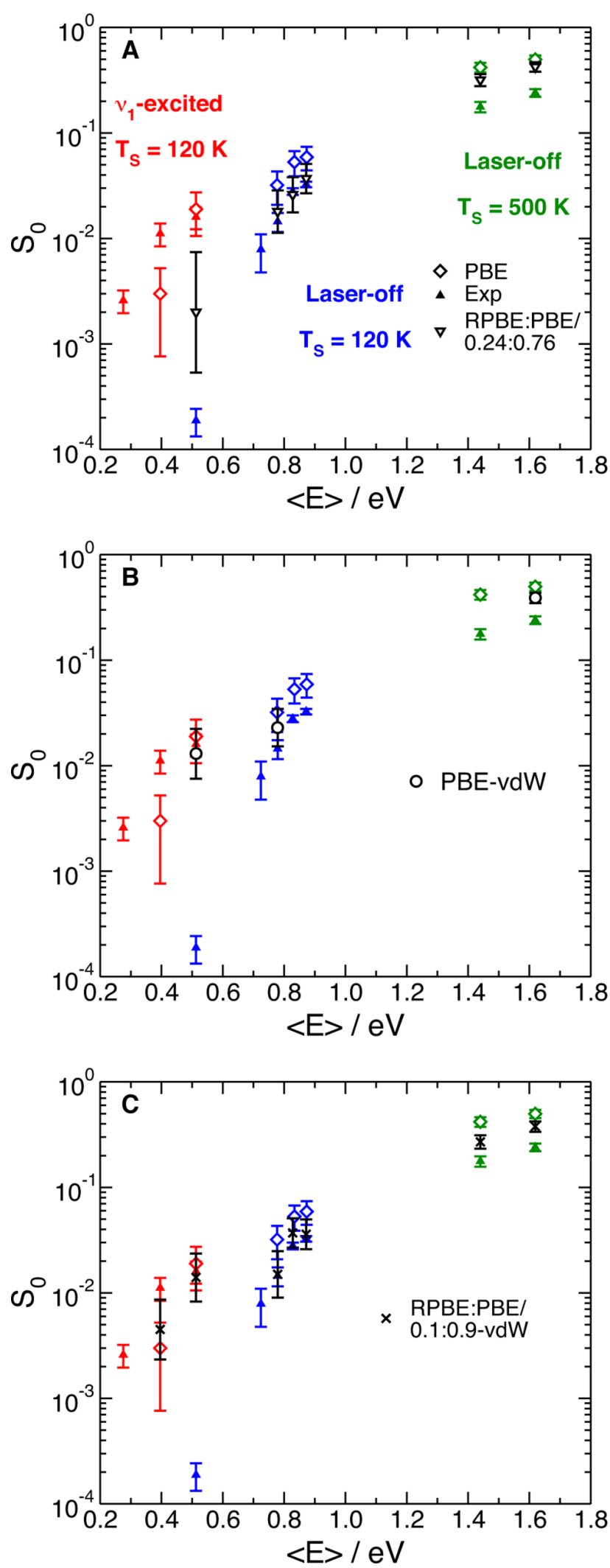

FIG. 3. Dissociation probability as a function of the average collision energy for $\mathrm{CHD}_{3}$ on $\mathrm{Pt}(111)$. Experimental data are compared to AIMD calculations performed with various exchange-correlation functionals. Error bars represent $95 \%$ confidence intervals in the AIMD data.

summarized with three points: (i) the PBE-AIMD calculations overestimate the experimental laser-off reaction probability; (ii) the PBE-AIMD laser-off reaction probability increases 
too steeply with increasing collision energy; (iii) the energy shift between the PBE-AIMD CH-stretch excited and laser-off reaction probability curves (i.e., the vibrational efficacy of the $\mathrm{CH}$-stretch mode) is too small compared to experiment.

In order to address point (i), we have first performed AIMD calculations using the RPBE:PBE/0.24:0.76 density functional. The energy shift between the PBE laser-off sticking probability points and the experimental data at the lowest collision energies simulated is about $0.1 \mathrm{eV}$, just like $0.1 \mathrm{eV}$ is the energy difference between the RPBE:PBE/0.24:0.76 and the PBE minimum barrier heights (see Section III B). Results are plotted in Figures 3(a) and 4(a), together with the PBEAIMD results and the experimental data. As expected from the higher energy barrier, the reaction probability computed with the RPBE:PBE/0.24:0.76 functional is lower than the PBE value for all collision energies. Very good agreement with experimental data is, therefore, obtained for the laser-off beams at the lowest collision energies (and $T_{s}=120 \mathrm{~K}$ ). The laser-off reactivity at the highest collision energies simulated (and $T_{s}=500 \mathrm{~K}$ ) is however only slightly lowered compared to the PBE results. The small relative change in $S_{0}$ is presumably due to the fact that the translational energy is so much in excess of the minimum energy barrier that the dynamics is not much affected by a $0.1 \mathrm{eV}$ difference in the barrier height. The use of the RPBE:PBE/0.24:0.76 functional, however, dramatically affects the reactivity of the $\mathrm{CH}$-stretch excited beams, and the computed reaction probability falls considerably below the experimental values when using this functional.

In Figure 5(a), we plot the projection of the center of mass on the surface unit cell for all the molecules that go on to react, as computed with the PBE functional for the largest collision energy considered $\left(\left\langle E_{i}\right\rangle=1.54 \mathrm{eV}\right)$. Reaction clearly occurs all over the surface unit cell and is not limited to the top site (i.e., the minimum energy barrier impact site ${ }^{48,49}$ ), in contrast to what was observed at lower collision energies. ${ }^{40}$ Consistently, the impact parameter distribution, i.e., the distribution of the lateral displacements between the center of mass of the reacting molecules and the closest first layer Pt atom, is very broad (Figure 5(c)). A possible explanation for the too steep increase in the PBE-AIMD reaction probability curve (point (ii)), which is not solved by the RPBE:PBE/0.24:0.76 density functional, could be that the PES corrugation is not correctly described by the PBE (and RPBE:PBE/0.24:0.76) functional: if the energy difference between the minimum barrier height and the barrier heights at other surface sites would be larger, the reaction probability would increase less rapidly with incidence energy.

Density functionals that account for dispersion interactions have recently been shown to produce broader reaction probability curves for $\mathrm{H}_{2}$ on $\mathrm{Ru}(0001){ }^{57}$ In order to verify whether this finding also applies to our system of interest, we have also calculated $S_{0}$ for $\mathrm{CHD}_{3}$ on $\mathrm{Pt}(111)$ using the PBE-vdW functional. Due to the computational cost of the AIMD method, we have computed only three sticking probability points using this functional. The three points chosen are representative of the laser-off reactivity on the large range of collision energies studied and of the two vibrational state populations considered. Interestingly, even though the minimum energy barrier calculated for the
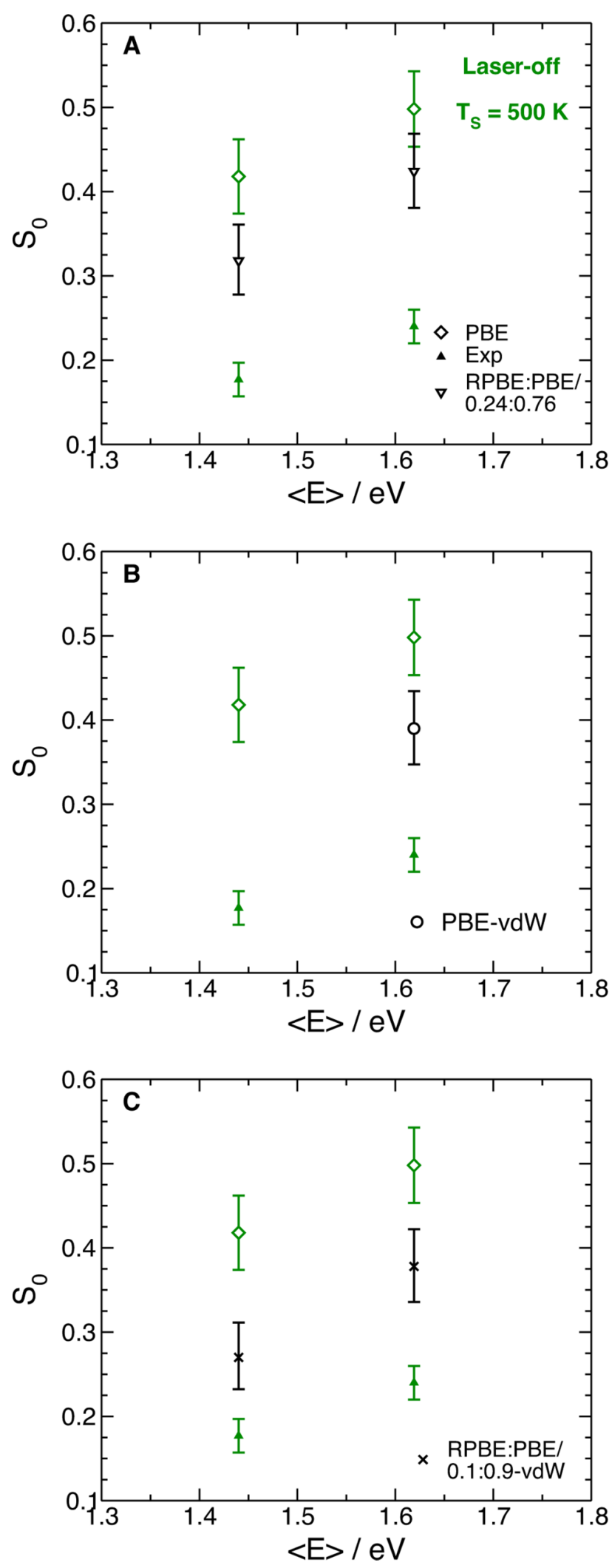

FIG. 4. Same as Figure 3, but the focus is on the highest energy points and the sticking probability is plotted on a linear scale. Error bars represent $95 \%$ confidence intervals in the AIMD data.

PBE-vdW functional is about $40 \mathrm{meV}$ lower than the PBE energy barrier (see Table III), the reaction probability calculated with the PBE-vdW functional is lower than for PBE in all cases (see Figures 3(b) and 4(b)). In particular, the 

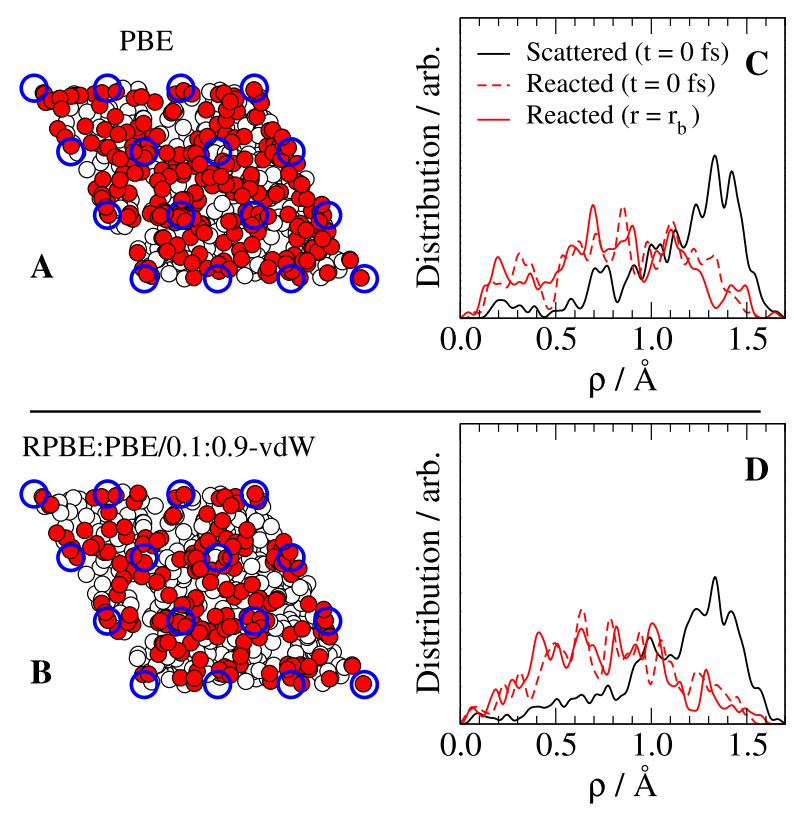

FIG. 5. (a) and (b) Bird-eye view of the initial center of mass positions of all the simulated molecules. Reacting and scattered molecules are plotted as red and white dots, respectively. The blue circles represent the first layer $\mathrm{Pt}$ atoms in their equilibrium positions. (c) and (d) Lateral displacement distributions calculated between the center of mass of the molecule and the closest first layer Pt atom (impact parameter $\rho$ ). The initial distribution and the distribution calculated at the transition state for the reacting molecules are plotted as a dashed and a solid red line, respectively. The initial distribution calculated for scattered molecules is plotted as a solid black line. All data refer to $\mathrm{CHD}_{3}$ on $\operatorname{Pt}(111)$ at $T_{s}=500 \mathrm{~K}$ and $\left\langle E_{i}\right\rangle=1.54 \mathrm{eV}$.

PBE-vdW laser-off reactivity agrees with the experimental data at the lowest collision energy simulated to within the experimental and calculated error bars, and $S_{0}$ is decreased with respect to the PBE value by about $10 \%$ at the highest collision energy. The reactivity obtained simulating a $\mathrm{CH}-$ stretch excited molecular beam is only slightly affected, and the agreement with experimental data is still good. This clearly shows that replacing PBE with vdW correlation improves the description of reactive scattering of $\mathrm{CHD}_{3}$ from $\mathrm{Pt}(111)$.

If we use an exchange functional of the form of Equation (1), with a mixing coefficient $x=0.1$ in combination with the vdW correlation functional (the RPBE:PBE/0.1:0.9$\mathrm{vdW}$ functional), we obtain a slightly lower laser-off reactivity compared to the PBE-vdW functional (see Figure 3(c)), which is consistent with a slightly higher dissociation barrier for the former (see Table III). The agreement with the experimental data is thereby further improved. At the highest collision energies considered, theory still overestimates the experimental data (see Figure 4(c)), but the discrepancy is considerably reduced with respect to the PBE calculations.

The RPBE:PBE/0.1:0.9-vdW functional returns as broad an impact parameter distributions as the PBE functional (the distribution for $\left\langle E_{i}\right\rangle=1.54 \mathrm{eV}$ is shown in Figure 5(d)). Therefore, the reason why the PBE-vdW and the RPBE:PBE/0.1:0.9-vdW functionals return a lower reactivity than the PBE functional even though the barrier heights of these functionals are equal to or lower than the PBE barrier height does not to lie in an increased PES corrugation in the degrees of freedom corresponding to the lateral motion of the molecule ( $X$ and $Y$ ). Similarly, $\theta$ and $\phi$ distributions computed with the PBE and the RPBE:PBE/0.1:0.9-vdW functionals for the reacting molecules are equally broad (not shown). Our results, therefore, suggest that the reason for the lower reactivity of the RPBE:PBE/0.1:0.9-vdW functional lies in a somewhat more difficult access to the TS that could be due to the more elongated $\mathrm{CH}$ bond at the TS (see Table IV). Most notably, by using the RPBE:PBE/0.1:0.9-vdW functional, we obtain a good description of the reactivity for the $\mathrm{CH}$ stretch excited beams. Considering the improved agreement for both laser-off and $\mathrm{CH}$-stretch excited conditions, we conclude that the proposed functional better describes the vibrational efficacy of the $\mathrm{CH}$-stretch mode than the $\mathrm{PBE}$ functional (point (iii)). An explanation for this is that the TS is slightly "later," since the dissociating $\mathrm{CH}$ bond is $50 \mathrm{~m} \AA$ more elongated than with the PBE functional. According to Polanyi's rules ${ }^{60}$ vibrational excitation should then be more effective in enhancing reactivity.

Summarizing, the RPBE:PBE/0.1:0.9-vdW functional has been found to generally improve the description of the $\mathrm{CHD}_{3}$ reactivity on $\operatorname{Pt}(111)$ over the PBE functional: a lower reactivity is obtained in the simulation of laser-off beams (point (i)), a smaller increase in reactivity is observed over the energy range for which laser-off experimental data are available (point (ii)), and a larger vibrational efficacy is found for the $\mathrm{CH}$-stretch mode of $\mathrm{CHD}_{3}$ (point (iii)). The laser-off reaction probability curve, however, still increases too steeply with increasing collision energy, such that the experimental laser-off reaction probability is overestimated at the highest collision energies considered. Considering this discrepancy still present between theory and experiments, we consider the aim of obtaining a SRP density functional for methane reacting on $\operatorname{Pt}(111)$ not yet achieved with the RPBE:PBE/0.1:0.9-vdW functional. If we assume that the AIMD method also correctly describes the laser-off reactivity of the hotter nozzle beams, our work suggests that a good functional for this system should exhibit a larger energetic corrugation than the PBE and the RPBE:PBE/0.1:0.9-vdW functionals. Other functionals that account for the vdW interaction, like the vdW-DF $2,{ }^{91}$ could be tested for this purpose.

As a final note, we mention again that the level of agreement obtained with experimental data using AIMD in combination with the RPBE:PBE/0.1:0.9-vdW functional is expected to depend on the computational setup employed in the sense that this functional would return a barrier about $45 \mathrm{meV}$ lower when using a larger amount of vacuum space (see Section III A).

\section{Surface temperature dependence of $S_{0}$}

Using the functional that returned the best agreement with the quantum-state resolved experiments for $\mathrm{CHD}_{3}$ on $\operatorname{Pt}(111)$, i.e., the RPBE:PBE/0.1:0.9-vdW functional, we have investigated the dependence of the methane dissociation reaction on $T_{s}$. We have considered $\mathrm{CH}_{4}$ as various groups measured the dissociation probability as a function of the surface temperature for this isotopologue, ${ }^{9,11,50,51,53,92}$ whereas, to our knowledge, no such data exist for $\mathrm{CHD}_{3}$. This, however, is not much of a concern as here we do not 
aim at determining vibrational mode specific observables, for which classical mechanics is expected to be less accurate when multiple vibrational modes with similar frequencies are present (as in $\mathrm{CH}_{4}$ ).

In simulating the surface temperature dependence of the methane dissociation reaction, we have considered data reported by Luntz and Bethune ${ }^{9}$ who measured dissociation probabilities over a particularly wide range of surface temperatures. Although the largest relative enhancement of the dissociation probability with increasing surface temperature has been observed ${ }^{9,50,51}$ and predicted ${ }^{16,26,34}$ for low collision energies, we have focused here on high collision energies such that the associated absolute dissociation probabilities are large enough for the AIMD method to be applied with statistical significance. The collision energy $E_{i}=1.27 \mathrm{eV}$ has been estimated by the authors to correspond to the maximum of the time-of-flight spectra recorded for the molecular beams, while the spread of the collision energy distribution has been reported to be $10 \%-20 \%$ of $E_{i} .{ }^{9}$ For this collision energy, dissociation probabilities are available in the range $T_{s}=400 \mathrm{~K}$ to $T_{s}=1300 \mathrm{~K}$. We have (linearly) extrapolated the dissociation probability as a function of $T_{S}$ down to $T_{s}=120 \mathrm{~K}$ (see Figure 6). Explorative calculations performed at the collision energy of $E_{i}=1.27 \mathrm{eV}$ for $T_{s}=120 \mathrm{~K}$ returned a reaction probability too high compared to the experimental data (not shown). This is consistent with the overestimation of the experimental reaction probability observed also for $\mathrm{CHD}_{3}$ at the highest collision energies (Section III C). However, we consider the comparison between theory and experiment on the $T_{s}$ dependence of the reaction to be most fair if it is made for similar values of reaction probability (rather than for similar collision energies).

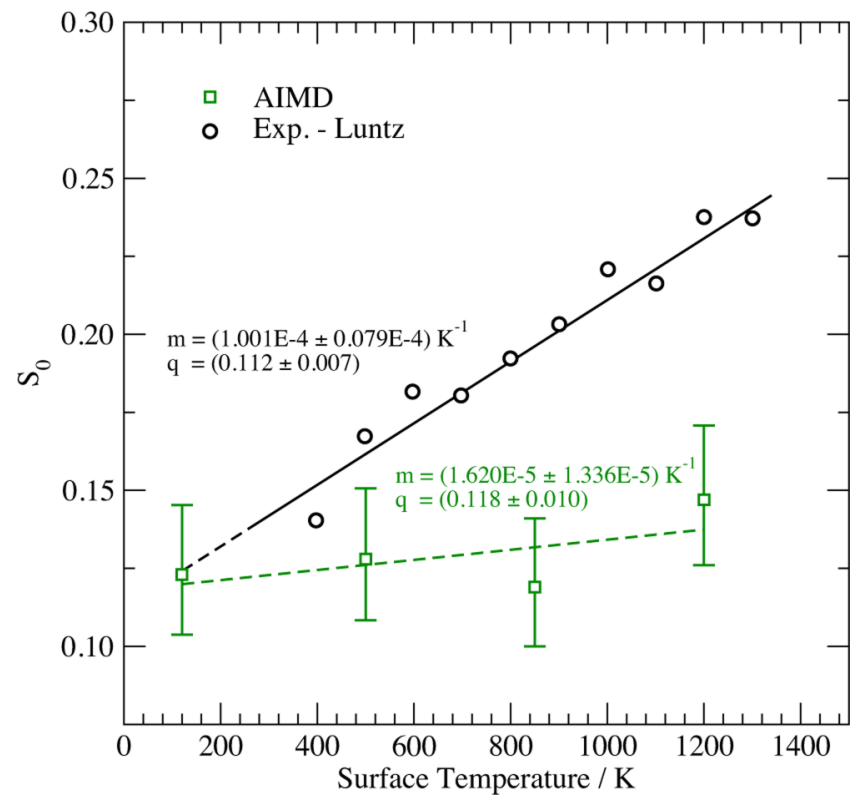

FIG. 6. Dissociation probability as a function of the surface temperature for $\mathrm{CH}_{4}$ on $\mathrm{Pt}(111)$. AIMD calculations performed with the RPBE:PBE/0.1:0.9$\mathrm{vdW}$ functional (green squares) are compared to experimental data from Ref. 9 (black circles). Lines represent linear fits of the AIMD and of the experimental data.
We have therefore modeled a translational energy distribution with average collision energy equal to $1.06 \mathrm{eV}$, which produced almost the same reaction probability as the linear fit to the experimental data extrapolated to $T_{s}=120 \mathrm{~K}$. To model the beam velocity spread, we have used a stream velocity parameter $v_{s}=3556 \mathrm{~m} / \mathrm{s}$ and a translational temperature relative to the average collision energy equal to $25 \mathrm{~K}$, as also used by Donald et $a .^{27}$ for modeling the translational energy distributions for the experiments of Luntz and Bethune. ${ }^{9}$ This value, in fact, returns a translational energy dispersion in agreement with the 10\%-20\% range reported in Ref. 9 and corresponds to a width parameter for the velocity distribution, $\alpha$, of $161 \mathrm{~m} / \mathrm{s}$. Regarding the vibrational energy content of the beam, we have modeled a Boltzmann distribution of vibrational states according to the experimental nozzle temperature (as done for the laser-off beam simulations in Section III C). At the considered nozzle temperature $\left(T_{n}=680 \mathrm{~K}\right.$ ), most of the $\mathrm{CH}_{4}$ molecules (about $76 \%$ ) are expected to be in the vibrational ground state.

We have calculated $S_{0}$ for four $T_{s}$ values, in the range $120 \mathrm{~K} \leqslant T_{s} \leqslant 1200 \mathrm{~K}$. Table $\mathrm{V}$ reports the number of differently initialized surface equilibration runs from which the surface initial conditions have been taken in the moleculesurface simulations and, for each of the initially imposed $T_{s}$ 's, the average surface temperatures $\left\langle T_{s}\right\rangle$ computed.

The motion of the surface atoms towards the molecule is known to significantly lower the dissociation barrier. ${ }^{46-49}$ In order to verify whether we sample a suitable range of vertical displacements of the first layer atoms in our model, we have determined the root mean square displacements of first layer surface atoms in the direction perpendicular to the surface $\left\langle u_{z, 1}^{2}\right\rangle^{\frac{1}{2}}$ in the equilibrated slabs and compared with available experimental data. ${ }^{93-95}$ The mean square displacements have been calculated as

$$
\left\langle u_{\alpha, 1}^{2}\right\rangle=\frac{1}{N_{j}} \sum_{j}\left(\left\langle x_{\alpha, j}^{2}\right\rangle-\left\langle x_{\alpha, j}\right\rangle^{2}\right),
$$

where $x_{\alpha, j}$ is the $\alpha$ th Cartesian coordinate of the $j$ th atom in the first layer, $N_{j}$ is the number of (equivalent) atoms in the first layer of the slab considered in the averaging, and the angle brackets represent time averages. The calculated values of $\left\langle u_{z, 1}^{2}\right\rangle^{\frac{1}{2}}$ are reported in Table VI for the simulated surface temperatures. Note that $\left\langle u_{z, 1}^{2}\right\rangle^{\frac{1}{2}}$ clearly increases when increasing surface temperature. The experimental range of root mean square displacements in the direction perpendicular to the surface, as calculated from the available experimental surface Debye temperature values (for the $z$ component

TABLE V. Number of surfaces used to sample the surface initial conditions for each of the initially imposed $T_{S}$ (in $\mathrm{K}$ ), and average surface temperatures $\left\langle T_{s}\right\rangle$ computed (in $\mathrm{K}$ ) with standard deviations.

\begin{tabular}{lcc}
\hline \hline Imposed $T_{S}$ & No. of surfaces & $\left\langle T_{s}\right\rangle \pm \sigma$ \\
\hline 120 & 10 & $127 \pm 17$ \\
500 & 10 & $485 \pm 65$ \\
850 & 9 & $893 \pm 114$ \\
1200 & 12 & $1228 \pm 166$ \\
\hline \hline
\end{tabular}


TABLE VI. Root mean square amplitudes (in $\AA$ ) along $z$ for the first layer atoms $\left\langle u_{z, 1}^{2}\right\rangle^{\frac{1}{2}}$ for the surfaces equilibrated at a given $T_{s}$ (in $\mathrm{K}$ ). The experimental ranges have been calculated from the available measured values of the $z$-component of the surface Debye temperatures. ${ }^{93-95}$

\begin{tabular}{lcc}
\hline \hline$T_{s}$ & AIMD & Exp. range \\
\hline 120 & 0.070 & $0.065-0.085$ \\
500 & 0.131 & $0.133-0.174$ \\
850 & 0.177 & $0.174-0.227$ \\
1200 & 0.233 & $0.206-0.270$ \\
\hline \hline
\end{tabular}

only), ${ }^{93-95}$ is also reported in Table VI. The values of $\left\langle u_{z, 1}^{2}\right\rangle^{\frac{1}{2}}$ as computed from the AIMD clean-surface equilibration runs are in reasonable agreement with these experimental ranges, validating the way in which the vertical displacements of the first layer surface atoms are sampled in our dynamical model.

The results are presented in Figure 6, where the dissociation probabilities are plotted as a function of the initially imposed surface temperature. Figure 6 also shows linear fits of both the experimental data from Ref. 9 and the theoretical data. Despite the large increase in reactivity observed in the experiment by Luntz and Bethune, only a moderate increase of the reactivity with $T_{s}$ is found with AIMD: the slope in the linear fits (also reported in Figure 6) is about six times larger for experiment than for theory.

The reason that our model does not describe the $T_{s}$ dependence of the reaction probability as determined by Luntz and Bethune ${ }^{9}$ is not yet clear. On the one hand, the AIMD method has already been found ${ }^{62,96}$ to underestimate the experimental surface temperature dependence of the dissociation probability of $\mathrm{D}_{2}$ on $\mathrm{Cu}(111) .{ }^{97}$ On the other hand, the extent to which surface temperature enhances reactivity has been experimentally observed to decrease with increasing collision energy, ${ }^{9,50,51}$ and Schoofs et al. ${ }^{92}$ even reported that no dependence of the sticking probability on $T_{S}$ was observed (within their experimental sensitivity ${ }^{98}$ ) at $E_{i} \approx 0.7 \mathrm{eV}$ (about $0.3 \mathrm{eV}$ lower than the collision energy that we have simulated). Moreover, a recent theoretical study even predicted a decrease of the dissociation probability which increasing surface temperature above a certain collision energy. ${ }^{32}$ Further experimental investigation on the $T_{s}$-dependence of the dissociation probability for methane reacting on $\operatorname{Pt}(111)$ at high collision energies is, therefore, advocated.

\section{E. Rotational alignment dependence of $S_{0}$}

As for the dependence of the methane dissociation reaction on $T_{s}$, we have employed the RPBE:PBE/0.1:0.9-vdW functional, which returns the best agreement with the quantum-state resolved experiments for $\mathrm{CHD}_{3}$ on $\mathrm{Pt}(111)$, for studying the rotational alignment dependence of the sticking probability. Experimental data concerning the rotational alignment dependence of methane dissociating on metal surfaces are available for the $\mathrm{CHD}_{3}+\mathrm{Ni}(100)$ system $^{19}$ and for the reaction of $\mathrm{CH}_{4}$ on $\mathrm{Ni}(100),{ }^{19} \mathrm{Ni}(110), \mathrm{Ni}(111)$, and $\mathrm{Pt}(111){ }^{38}$ In these experiments, linearly polarized light has been used to selectively excite a specific vibrational mode of the molecule ( $v_{1}$ for $\mathrm{CHD}_{3}$ and $v_{3}$ for $\mathrm{CH}_{4}$ ) and the selection rules of the IR-active transition employed have been used to generate rotationally aligned molecular beams. For transitions in the R-branch $(\Delta J=+1)$, for instance, the $\Delta M=0$ and $\Delta K=0$ selection rules allow $\mathrm{CHD}_{3}$ molecules initially in the rotational ground state to be exclusively excited (in the laser polarization reference frame) to the $J=1, M=0, K=0$ rotational sublevel of the $v_{1}=1$ vibrational state (and not to the $M= \pm 1$ sublevels). This particular transition, which is generally labeled as ${ }^{0} \mathrm{R}(0)$, produces the strongest alignment for both the angular momentum ${ }^{99}$ and the vibrational transition dipole moment. ${ }^{100} \mathrm{By}$ varying the polarization direction of the laser, different angular momentum and vibrational transition dipole moment alignments can be obtained. The difference in reactivity between the laser being polarized in the direction parallel to the surface and perpendicular to the surface has been expressed by the experimentalists in terms of the alignment contrast $\Delta p$, defined as $\Delta p=\frac{S_{0}^{\|}-S_{0}^{\perp}}{S_{0}^{\|}+S_{0}^{\perp}}$, where $S_{0}^{\|}$and $S_{0}^{\perp}$ are the sticking probabilities for the two laser polarization directions.

In the simulation of this type of experiments with AIMD, the choice of the $\mathrm{CHD}_{3}$ isotopologue of methane is preferred (rather than $\mathrm{CH}_{4}$ ) to minimize the risk that artificial IVR induced by the use of classical mechanics ${ }^{101}$ might affect the computed vibrational-mode specific reaction probabilities. To our knowledge, the only experiments on the rotational alignment dependence of the $\mathrm{CHD}_{3}$ dissociation reaction have been performed on $\mathrm{Ni}$ surfaces, ${ }^{19,39}$ but similar effects are expected to characterize the reaction of $\mathrm{CHD}_{3}$ on $\mathrm{Pt}(111)$, as the alignment contrast $\Delta p$ has been reported ${ }^{38}$ to be similar for $\mathrm{CH}_{4}$ reacting on $\operatorname{Pt}(111)$ and on $\mathrm{Ni}$ surfaces. Once again, most of the experiments have been performed for low collision energies $\left(E_{i} \approx 0.3 \mathrm{eV}\right)$. Such conditions cannot be simulated with AIMD, because the computational cost of the AIMD trajectories presently rules out an accurate estimate of reaction probabilities lower than $1 \%$. In addition, the QCT method implemented here is not suitable for dealing with total (vibrational + translational) energies which are close to (or lower than) the minimum energy barrier, as the possibility that tunneling effects may play a role at the low collision energies used in the experiments cannot be completely excluded.

We have therefore performed calculations for larger collision energies, investigating the effect that rotational alignment might have for energies at which experimental data are not yet available. We have sampled a translational energy distribution also sampled in the calculation of one sticking probability point for laser-off conditions (the point that corresponds to the molecular beam experiment with $\left\langle E_{i}\right\rangle=0.83 \mathrm{eV}$ and $T_{\text {gas }}=902 \mathrm{~K}$, see Section III C; see also Ref. 40 for the translational energy distribution parameters). A surface temperature of $120 \mathrm{~K}$ has been simulated in these calculations.

We have investigated the effect on the reactivity of the excitation of the $v_{1}$ mode through the ${ }^{0} \mathrm{R}(0)$ transition for $\mathrm{CHD}_{3}$ for two rotationally aligned states. We have simulated molecules initially in the $v_{1}=1, J=1, K=0$ rovibrational state, with the $M$ quantum number either set equal to 0 or equal to 1 . With the space fixed axis arbitrarily chosen to lay along the surface normal (see also Section II), light polarized 
in the direction perpendicular to the surface selectively excites molecules to the $M=0$ state, while light polarization in the direction parallel to the surface excites molecules in a mixture of $M=1$ and $M=-1$ states. In fact, the ${ }^{0} \mathrm{R}(0)$ transition excites molecules exclusively to the $J=1, K=0, M=0$ state if the laser polarization reference frame is considered, while various $M$ states have to be considered if a different reference frame is assumed. We always chose our reference frame with the space-fixed axis lying along the surface normal. If we assume only the angle $\chi$ between the surface normal and the laser polarization direction to be relevant for the purpose of the alignment effect investigated here (and not, for instance, the angle formed with specific surface crystallographic directions), then the initial rotational state $\Psi_{0}$ of a molecule can be expressed as

$$
\Psi_{0}=\sum_{M=-1}^{+1} d_{K=0, M}^{J=1}(\chi) \Phi_{J=1, K=0, M},
$$

where the $\Phi_{J, K, M}$ terms are the rotational eigenfunctions of the Hamiltonian for a symmetric (rigid) rotor as defined in the reference frame where the space-fixed axis is the surface normal, and the $d_{K, M}^{J}(\chi)$ coefficients are the so-called Wigner (small) $d$-functions. ${ }^{102}$ By explicitly writing the expression of the $d$-functions in Equation (3), we obtain the following:

$\Psi_{0}=-\frac{1}{\sqrt{2}} \sin \chi \Phi_{1,0,-1}+\cos \chi \Phi_{1,0,0}+\frac{1}{\sqrt{2}} \sin \chi \Phi_{1,0,1}$.

Therefore, using the reference frame with the surface normal as quantization axis, the ${ }^{0} \mathrm{R}(0)$ transition populates only the $M=0$ state for a laser polarization direction perpendicular to the surface $\left(\chi=0^{\circ}\right)$, while a superposition of the $M=-1$ and $M=1$ states is obtained with the same transition for a laser polarization direction parallel to the surface $\left(\chi=90^{\circ}\right)$. Alternatively, if the laser polarization reference frame was assumed, only the $M=0$ state would have to be considered, and the space-fixed axis would then have to be rotated to simulate the different laser polarization directions, once again setting up only the $M=0$ state with respect to the quantization axis thus defined.

The alignment of the angular momentum is then strictly related to the alignment of the figure axis of the molecule (and therefore to the $\mathrm{CH}$ bond orientation). Despite the fact that the angular momentum distribution is different (symmetric with respect to the origin of the $z$ axis) for molecules in the $M=1$ state and in the $M=-1$ state, the figure axis distribution is identical for these two rotational sublevels. Assuming the figure axis ( $\mathrm{CH}$ bond) alignment effect on the reactivity to dominate over the angular momentum effect (as also observed experimentally ${ }^{19,38}$ ), we have only simulated molecules in the $M=1$ state as representative for the experimental conditions with the laser oriented in the direction parallel to the surface. In the classical picture, the figure axis distributions obtained when preparing molecules in the $v_{1}=1, J=1, K=0, M= \pm 1$ and $v_{1}=1, J=1, K=0, M=0$ states are illustrated in Figure 7: For $M= \pm 1$, we have the highest chance of finding the figure axis forming an angle $\beta=45^{\circ}$ (or, equivalently, $\beta=135^{\circ}$ ) with the surface normal, while the probability of finding the figure axis oriented
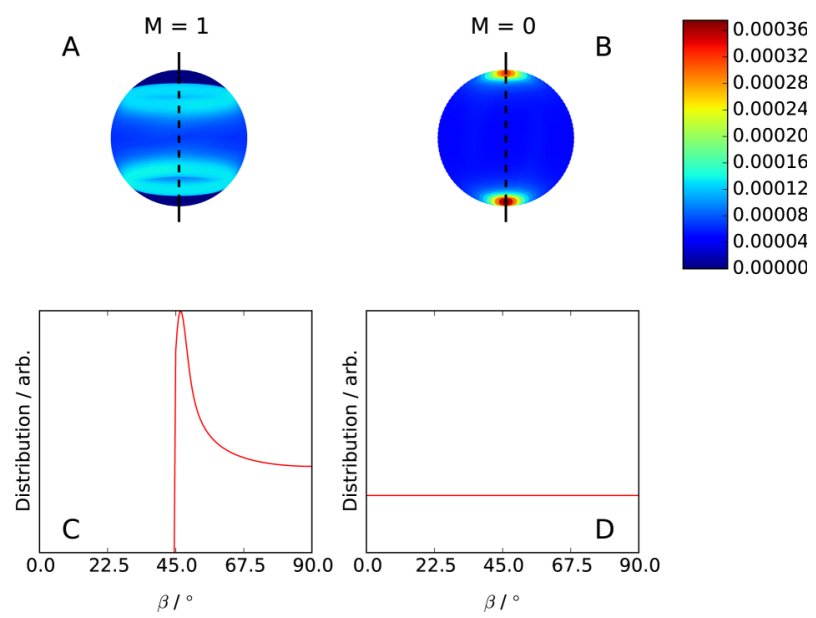

FIG. 7. Figure axis ( $\mathrm{CH}$ bond) orientation distributions in the simulations of rotationally aligned $\left(v_{1}=1, J=1, K=0\right)$ states. ((a) and (b)) Probability density plotted on a spherical surface. The red (dark blue) areas correspond to the solid angles where the probability density of having the figure axis oriented is highest (lowest). The $z$-axis is also represented as a black line. ((c) and (d)) Corresponding $\beta$ (polar angle) distributions, obtained integrating the distributions shown in (a) and (c) over the azimuthal angle. (a) and (c) refer to the $M=1$ state, (b) and (d) to the $M=0$ state. The distributions for $M=-1$ are identical to the $M=1$ distributions.

perpendicular to the surface is zero (as it is in the whole range $\beta<45^{\circ}$ and $\beta>135^{\circ}$ ). For $M=0$, on the other hand, the orientation distribution initially sampled by the figure axis is uniform in $\beta$ (Figure 7(d)), which means that the probability density per solid angle is the largest for orientations of the figure axis towards (and away from) the surface, while it is the lowest for orientations parallel to the surface (Figure 7(b)).

The reaction probability values calculated for the $v_{1}=1, J=1, K=0, M=0$ and $v_{1}=1, J=1, K=0, M=1$ states as well as the reaction probability calculated simulating laser-off conditions (i.e., sampling a Boltzmann distribution of vibrational states) are presented in Figure 8. No effect of the

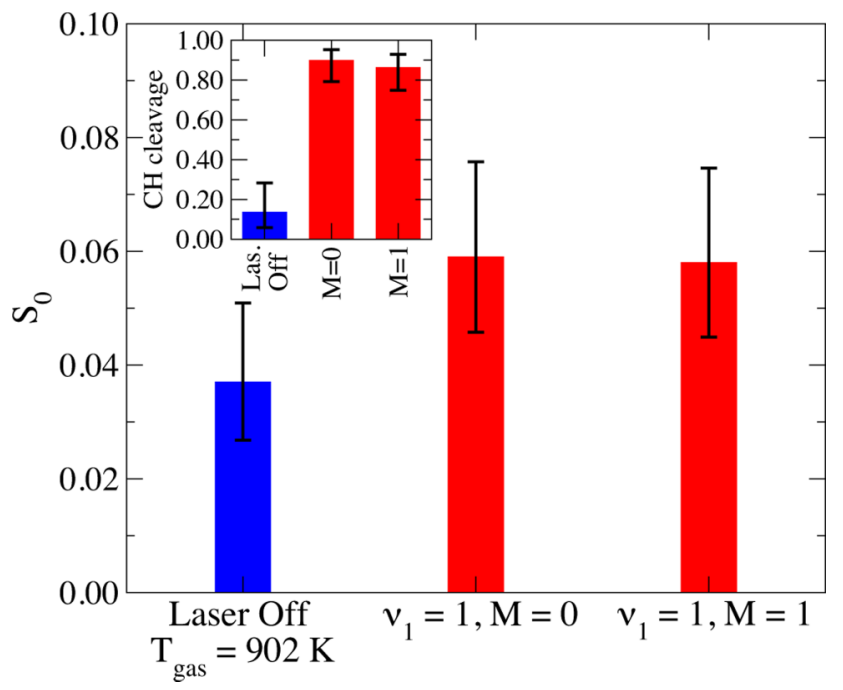

FIG. 8. Reaction probability obtained simulating laser-off conditions or simulating rotationally aligned states $\left(v_{1}=1, J=1, K=0, M=0,1\right)$. The RPBE:PBE/0.1:0.9-vdW functional has been employed here. The average collision energy is $0.83 \mathrm{eV}$ and the surface temperature is $120 \mathrm{~K}$. In the inset, the fraction of $\mathrm{CH}$ dissociation in the reacting molecules for the three conditions is shown. 
rotational alignment has been detected within our statistical accuracy: the reactivity of the $J=1, K=0, M=0$ and the $J=1, K=0, M=1$ rotational sublevels of the $v_{1}=1$ state has been found to be essentially the same. This result might have to do with the collision energy at which our calculations have been performed: at low collision energy, where only few molecules can react, changing the rotational alignment (and therefore the most sampled orientation) of the molecules has been observed to considerably affect the reactivity, ${ }^{19,38}$ while at larger collision energies, where more paths become available for the reaction, the rotational alignment dependence of the reaction might become weaker (or non-existent), as also observed for the dissociation of $\mathrm{D}_{2}$ on $\mathrm{Cu}(111) .{ }^{62,103}$ This would be consistent with the fact that the alignment effect for $\mathrm{CHD}_{3}$ on $\mathrm{Ni}(100)$ has been observed to become weaker with increasing collision energy: when going from $E_{i}=0.31 \mathrm{eV}$ to $E_{i}=0.73 \mathrm{eV}$ the value of $\Delta p$ decreases from 0.2 to about $0.1 .^{39}$ The reactivity obtained simulating molecules in the $v_{1}=1$ vibrational state is considerably larger (a factor 1.6) than the reactivity obtained averaging over all the vibrational states populated through thermal excitations. This is consistent with the large vibrational efficacy observed for the $\mathrm{CH}$-stretch $\left(v_{1}\right)$ mode of $\mathrm{CHD}_{3}$. The excitation of the $v_{1}$ mode is also accompanied by a significant change in the fraction of molecules that dissociate through $\mathrm{CH}$ bond cleavage (see inset in Figure 8): a majority of $\mathrm{CD}$ bond breaking is observed when simulating laser-off conditions, while the $\mathrm{CH}$ bond breaking becomes dominant when considering $\mathrm{CH}$ stretch excited molecules. Note that at the collision energy considered, the preference for $v_{1}$ excited molecules to dissociate through breaking of the $\mathrm{CH}$ bond is not absolute, as CD bonds also dissociate. This is consistent with enough (translational) energy being available for the dissociation of the CD bonds as well. Also, previous work has shown how the bond selective character of the dissociation of $\mathrm{CHD}_{3}\left(v_{1}=1\right)$ on metal surfaces is gradually lost with increasing collision energy. ${ }^{25,31,32,89}$

\section{CONCLUSIONS AND OUTLOOK}

We have tested the influence of the exchange-correlation functional $E_{X C}$ on the reactivity of methane on a platinum surface, using the dissociation of the $\mathrm{CHD}_{3}$ isotopologue on $\operatorname{Pt}(111)$ as a test case. Dynamical calculations have been performed using the AIMD technique, which is suitable for the purpose of testing the influence of $E_{X C}$ on the sticking probability. In fact, the AIMD method allows the calculation of statistically accurate dissociation probabilities without the need of introducing a priori dynamical approximations concerning the evolution of specific molecular degrees of freedom or concerning the role played by surface atom motion. The sticking probabilities computed here have been compared to recent quantum state-resolved experimental data and to previous calculations performed using the PBE density functional. ${ }^{40}$

The calculations reveal that a density functional that returns a minimum barrier height $0.1 \mathrm{eV}$ higher than the PBE functional (RPBE:PBE/0.24:0.76) improves the agreement with laser-off experimental data at the lowest collision energies simulated, but only slight improvement is observed at the high collision energies, and the agreement is drastically worsened for $\mathrm{CH}$-stretch excited beams. The overall best agreement is observed for a density functional consisting of the correlation functional of Dion et al.,${ }^{56}$ developed to mimic the vdW interaction, and a linear combination of the RPBE and PBE exchange functionals (the RPBE:PBE/0.1:0.9-vdW functional). This functional improves the agreement with experimental data with respect to the previous PBE calculations in three ways. First, the reactivity of the simulated laser-off beams is lowered, both at the lowest and at the highest collision energies investigated. Interestingly, the barrier height that this functional returns is the same as for the PBE functional, so that the lowering of the laser-off reactivity predicted by the RPBE:PBE/0.1:0.9-vdW functional is due to other aspects of the PES. Second, the laser-off reaction probability curve increases more gradually (less steeply) over the collision energy range for which experiments are available. This functional, however, still returns a somewhat too high reaction probability when simulating the high collision energy laser-off molecular beams. In this respect, functionals yielding interaction energies characterized by a larger corrugation in the $X$ and $Y$ degrees of freedom are expected to return lower reactivity at the highest collision energies simulated, improving the agreement with experimental data. Functionals that account for the van der Waals interaction through a different correlation functional ${ }^{91}$ might be tested to this end. Third, the vibrational efficacy obtained for the $\mathrm{CH}$-stretch mode is increased, as the agreement with $v_{1^{-}}$ excited experiments remains good. The improved (larger) vibrational efficacy of the $\mathrm{CH}$-stretch mode obtained with the RPBE:PBE/0.1:0.9-vdW functional is most likely due to the more elongated dissociating $\mathrm{CH}$ bond at the TS obtained with this functional.

By using the RPBE:PBE/0.1:0.9-vdW functional, we have also investigated the surface temperature dependence of the dissociation of $\mathrm{CH}_{4}$ on $\mathrm{Pt}(111)$, comparing our calculations to experimental data. ${ }^{9}$ A modest increase of the sticking probability with surface temperature has been observed, but the effect observed is smaller than that observed by Luntz and Bethune. ${ }^{9}$ With the same RPBE:PBE/0.1:0.9-vdW functional, we have also looked at the effect of the rotational alignment on the reactivity of $\mathrm{CHD}_{3}$ on $\mathrm{Pt}(111)$, at collision energies at which statistical accurate reaction probabilities could be determined with AIMD $\left(\left\langle E_{i}\right\rangle=0.83 \mathrm{eV}\right)$. The simulation of $\mathrm{CHD}_{3}$ in the $M=0$ and $M=1$ sublevels of the $v_{1}=1, J=1, K=0$ rovibrational state colliding with $\operatorname{Pt}(111)$ returned the same reaction probability, suggesting a minor effect of rotational alignment at the considered collision energy.

Finally, we consider ways in which a true SRP density functional may be derived for methane reacting on $\operatorname{Pt}(111)$, by making further improvements. One possible improvement is the use of a larger vacuum space between the periodic images of the slab combined with starting the molecule further away from the surface where its interaction with the surface is truly negligible. This would lead to the molecule hitting the surface with an increased collision energy, and keeping the agreement with the experimental data for the 
laser-off reactivity would require a higher percentage of the more repulsive RPBE exchange to be mixed in. This could lead to increased agreement with experiment for the vibrational efficacy, as the barrier would become slightly later. An alternative way of achieving this within the present (computationally cheaper) setup is to increase the molecule's initial velocity in accordance with the interaction it already has with the surface at a molecule-surface distance of $6 \AA$. Such a procedure can be justified on the basis of the dependence of the molecule-surface interaction being essentially independent of the molecular coordinates other than $Z$, for $Z \geqslant 6 \AA$.

In future studies, in developing a SRP density functional we suggest putting more emphasis on obtaining agreement with experiment for the $\mathrm{CH}$-stretch excited state over a larger range of energies than now done. The reason for this is that the quasi-classical approach should work well for this state: as argued and shown before by $\mathrm{us}^{40}$ and by others ${ }^{43,44}$ no artificial energy transfer will occur from the $\mathrm{CH}$-stretch to the other vibrational modes of $\mathrm{CHD}_{3}$, due to the isolated frequency of the $\mathrm{CH}$-stretch mode. For this reason, the vibrational efficacy of this mode for promoting the reaction compared to the vibrational ground state should be well described with quasi-classical mechanics. Furthermore, it might be better to restrict the comparison to experiments using high surface temperatures only. The experiments we compare to here that were done for low collision energies used a low surface temperature $(120 \mathrm{~K})^{40}$ so that the RAIRS detection technique ${ }^{104}$ could be used. ${ }^{24,33,73,105}$ However, reaction probabilities can also be measured at higher surface temperatures based on either Auger spectroscopy ${ }^{13,14,19,38,106}$ or the Kings and Wells technique. ${ }^{40,107,108}$ Using the same and a somewhat higher (than $120 \mathrm{~K}$ ) surface temperature for all experiments would ensure that in the comparison the AIMD calculations can be done for a surface temperature well above the Debye surface temperature of $\operatorname{Pt}(111)$ (in the range $111-143 \mathrm{~K}^{93-95}$ ), at which the motion of the surface atoms is probably better modeled with classical mechanics than at temperatures below or near the Debye surface temperature.

\section{ACKNOWLEDGMENTS}

The authors thank Rainer Beck and Bret Jackson for useful discussions. This work was made possible by financial support from the Nederlandse Organisatie voor Wetenschappelijk Onderzoek (Netherlands Organisation for Scientific Research, NWO) and from the European Research Council through an ERC-2013 advanced grant (No. 338580). NWO Exacte Wetenschappen, EW (NWO Physical Sciences Division) is acknowledged for granting access on the Cartesius supercomputer through a Dutch Computing Challenge Project (DCCP) (ID: SH-282-14). PRACE is also acknowledged for awarding access to the MareNostrum supercomputer based in Spain at the Barcelona Supercomputing Center (BSC) (ID: 2012071357).

${ }^{1}$ G. J. Kroes, Phys. Chem. Chem. Phys. 14, 14966 (2012).

${ }^{2}$ R. Peverati and D. G. Truhlar, Philos. Trans. R. Soc., A 372, 20120476 (2014).

${ }^{3}$ J. Zheng, Y. Zhao, and D. G. Truhlar, J. Chem. Theory Comput. 5, 808 (2009).
${ }^{4}$ C. Díaz, E. Pijper, R. A. Olsen, H. F. Busnengo, D. J. Auerbach, and G. J. Kroes, Science 326, 832 (2009).

${ }^{5}$ C. Díaz, R. A. Olsen, D. J. Auerbach, and G. J. Kroes, Phys. Chem. Chem. Phys. 12, 6499 (2010).

${ }^{6}$ L. Sementa, M. Wijzenbroek, B. J. van Kolck, M. F. Somers, A. Al-Halabi, H. F. Busnengo, R. A. Olsen, G. J. Kroes, M. Rutkowski, C. Thewes, N. F. Kleimeier, and H. Zacharias, J. Chem. Phys. 138, 044708 (2013).

${ }^{7}$ G. J. Kroes, J. Phys. Chem. Lett. 6, 4106 (2015).

${ }^{8}$ C. T. Rettner, H. E. Pfnür, and D. J. Auerbach, J. Chem. Phys. 84, 4163 (1986).

${ }^{9}$ A. C. Luntz and D. S. Bethune, J. Chem. Phys. 90, 1274 (1989).

${ }^{10}$ L. B. F. Juurlink, P. R. McCabe, R. R. Smith, C. L. DiCologero, and A. L. Utz, Phys. Rev. Lett. 83, 868 (1999).

${ }^{11}$ J. Higgins, A. Conjusteau, G. Scoles, and S. L. Bernasek, J. Chem. Phys. 114, 5277 (2001).

${ }^{12}$ L. Halonen, S. L. Bernasek, and D. J. Nesbitt, J. Chem. Phys. 115, 5611 (2001).

${ }^{13}$ R. D. Beck, P. Maroni, D. C. Papageorgopoulos, T. T. Dang, M. P. Schmid, and T. R. Rizzo, Science 302, 98 (2003).

${ }^{14}$ R. R. Smith, D. R. Killelea, D. F. DelSesto, and A. L. Utz, Science 304, 992 (2004).

${ }^{15}$ P. Maroni, D. C. Papageorgopoulos, M. Sacchi, T. T. Dang, R. D. Beck, and T. R. Rizzo, Phys. Rev. Lett. 94, 246104 (2005).

${ }^{16}$ S. Nave and B. Jackson, Phys. Rev. Lett. 98, 173003 (2007).

${ }^{17}$ D. R. Killelea, V. L. Campbell, N. S. Shuman, and A. L. Utz, Science 319, 790 (2008).

${ }^{18}$ L. B. F. Juurlink, D. R. Killelea, and A. L. Utz, Prog. Surf. Sci. 84, 69 (2009).

${ }^{19}$ B. L. Yoder, R. Bisson, and R. D. Beck, Science 329, 553 (2010).

${ }^{20}$ M. Sacchi, D. J. Wales, and S. J. Jenkins, J. Phys. Chem. C 115, 21832 (2011).

${ }^{21}$ B. Jackson and S. Nave, J. Chem. Phys. 135, 114701 (2011).

${ }^{22}$ M. Sacchi, D. J. Wales, and S. J. Jenkins, Phys. Chem. Chem. Phys. 14, 15879 (2012).

${ }^{23}$ M. Sacchi, D. J. Wales, and S. J. Jenkins, Comput. Theor. Chem. 990, 144 (2012).

${ }^{24}$ L. Chen, H. Ueta, R. Bisson, and R. D. Beck, Faraday Discuss. 157, 285 (2012).

${ }^{25}$ B. Jiang and H. Guo, J. Phys. Chem. C 117, 16127 (2013).

${ }^{26}$ B. Jiang, R. Liu, J. Li, D. Xie, M. Yang, and H. Guo, Chem. Sci. 4, 3249 (2013).

${ }^{27}$ S. B. Donald, J. K. Navin, and I. Harrison, J. Chem. Phys. 139, 214707 (2013).

${ }^{28}$ M. Mastromatteo and B. Jackson, J. Chem. Phys. 139, 194701 (2013).

${ }^{29}$ B. Jackson and S. Nave, J. Chem. Phys. 138, 174705 (2013).

${ }^{30}$ S. Nave, A. K. Tiwari, and B. Jackson, J. Phys. Chem. A 118, 9615 (2014),

${ }^{31}$ X. J. Shen, A. Lozano, W. Dong, H. F. Busnengo, and X. H. Yan, Phys. Rev. Lett. 112, 046101 (2014).

${ }^{32}$ A. Lozano, X. J. Shen, R. Moiraghi, W. Dong, and H. F. Busnengo, Surf. Sci. 640, 25 (2015).

${ }^{33}$ P. M. Hundt, H. Ueta, M. E. van Reijzen, B. Jiang, H. Guo, and R. D. Beck, J. Phys. Chem. A 119, 12442 (2015).

${ }^{34}$ X. Shen, Z. Zhang, and D. H. Zhang, Phys. Chem. Chem. Phys. 17, 25499 (2015).

${ }^{35}$ B. Jiang, M. Yang, D. Xie, and H. Guo, "Quantum dynamics of polyatomic dissociative chemisorption on transition metal surfaces: Mode specificity and bond selectivity," Chem. Soc. Rev. (published online).

${ }^{36}$ G. Jones, J. G. Jakobsen, S. S. Shim, J. Kleis, M. P. Andersson, J. Rossmeisl, F. Abild-Pedersen, T. Bligaard, S. Helveg, B. Hinnemann, J. R. RostrupNielsen, I. Chorkendorff, J. Sehested, and J. K. Nørskov, J. Catal. 259, 147 (2008).

${ }^{37}$ B. Ølgaard Nielsen, A. C. Luntz, P. M. Holmblad, and I. Chorkendorff, Catal. Lett. 32, 15 (1995).

${ }^{38}$ B. L. Yoder, R. Bisson, P. M. Hundt, and R. D. Beck, J. Chem. Phys. 135, 224703 (2011).

${ }^{39}$ B. L. Yoder, Steric Effects in the Chemisorption of Vibrationally Excited Methane on Nickel (Springer, Berlin, 2012).

${ }^{40}$ F. Nattino, H. Ueta, H. Chadwick, M. E. van Reijzen, R. D. Beck, B. Jackson, M. C. van Hemert, and G. J. Kroes, J. Phys. Chem. Lett. 5, 1294 (2014).

${ }^{41}$ J. P. Perdew, K. Burke, and M. Ernzerhof, Phys. Rev. Lett. 77, 3865 (1996).

${ }^{42}$ J. P. Perdew, K. Burke, and M. Ernzerhof, Phys. Rev. Lett. 78, 1396 (1997).

${ }^{43}$ G. Czakó and J. M. Bowman, J. Am. Chem. Soc. 131, 17534 (2009).

${ }^{44}$ G. Czakó and J. M. Bowman, Science 334, 343 (2011).

${ }^{45}$ B. Jackson, F. Nattino, and G. J. Kroes, J. Chem. Phys. 141, 054102 (2014). 
${ }^{46}$ A. K. Tiwari, S. Nave, and B. Jackson, J. Chem. Phys. 132, 134702 (2010).

${ }^{47}$ G. Henkelman and H. Jónsson, Phys. Rev. Lett. 86, 664 (2001).

${ }^{48}$ S. Nave and B. Jackson, J. Chem. Phys. 130, 054701 (2009).

${ }^{49}$ S. Nave, A. K. Tiwari, and B. Jackson, J. Chem. Phys. 132, 054705 (2010).

${ }^{50}$ J. Harris, J. Simon, A. C. Luntz, C. B. Mullins, and C. T. Rettner, Phys. Rev. Lett. 67, 652 (1991).

${ }^{51}$ D. J. Oakes, M. R. S. McCoustra, and M. A. Chesters, Faraday Discuss. 96, 325 (1993).

${ }^{52}$ P. M. Holmblad, J. Wambach, and I. Chorkendorff, J. Chem. Phys. 102, 8255 (1995).

${ }^{53}$ M. Valden, N. Xiang, J. Pere, and M. Pessa, Appl. Surf. Sci. 99, 83 (1996).

${ }^{54}$ Y. Y. Chuang, M. L. Radhakrishnan, P. L. Fast, C. J. Cramer, and D. G. Truhlar, J. Phys. Chem. A 103, 4893 (1999).

${ }^{55}$ A. Chakraborty, Y. Zhao, H. Lin, and D. G. Truhlar, J. Chem. Phys. 124, 044315 (2006)

${ }^{56}$ M. Dion, H. Rydberg, E. Schröder, D. C. Langreth, and B. I. Lundqvist, Phys. Rev. Lett. 92, 246401 (2004).

${ }^{57}$ M. Wijzenbroek and G. J. Kroes, J. Chem. Phys. 140, 084702 (2014).

${ }^{58}$ L. Martin-Gondre, J. I. Juaristi, M. Blanco-Rey, R. Díez Muiño, and M. Alducin, J. Chem. Phys. 142, 074704 (2015).

${ }^{59}$ B. Hammer, L. B. Hansen, and J. K. Nørskov, Phys. Rev. B 59, 7413 (1999).

${ }^{60}$ J. C. Polanyi, Acc. Chem. Rev. 5, 161 (1972).

${ }^{61}$ A. Groß and A. Dianat, Phys. Rev. Lett. 98, 206107 (2007).

${ }^{62}$ F. Nattino, C. Díaz, B. Jackson, and G. J. Kroes, Phys. Rev. Lett. 108, 236104 (2012)

${ }^{63}$ G. Kresse and D. Joubert, Phys. Rev. B 59, 1758 (1999).

${ }^{64}$ P. E. Blöchl, Phys. Rev. B 50, 17953 (1994).

${ }^{65}$ G. Kresse and J. Hafner, Phys. Rev. B 47, 558 (1993).

${ }^{66}$ G. Kresse and J. Hafner, Phys. Rev. B 49, 14251 (1994).

${ }^{67}$ G. Kresse and J. Furthmüller, Comput. Mater. Sci. 6, 15 (1996).

${ }^{68}$ G. Kresse and J. Furthmüller, Phys. Rev. B 54, 11169 (1996).

${ }^{69}$ J. Klimeš, D. R. Bowler, and A. Michaelides, Phys. Rev. B 83, 195131 (2011).

${ }^{70}$ J. W. Arblaster, Platinum Met. Rev. 41, 12 (1997).

${ }^{71}$ J. W. Arblaster, Platinum Met. Rev. 50, 118 (2006)

${ }^{72}$ H. A. Michelsen and D. J. Auerbach, J. Chem. Phys. 94, 7502 (1991).

${ }^{73}$ L. Chen, H. Ueta, H. Chadwick, and R. D. Beck, J. Phys. Chem. C 119, 14499 (2015).

${ }^{74}$ E. B. Wilson, J. Am. Stat. Assoc. 22, 209 (1927).

${ }^{75}$ W. L. Hase, Encyclopedia of Computational Chemistry (Wiley, New York, 1998).

${ }^{76}$ R. Liu, F. Wang, B. Jiang, G. Czakó, M. Yang, K. Liu, and H. Guo, J. Chem. Phys. 141, 074310 (2014).

${ }^{77}$ G. Román-Pérez and J. M. Soler, Phys. Rev. Lett. 103, 096102 (2009).

${ }^{78}$ Y. Zhang and W. Yang, Phys. Rev. Lett. 80, 890 (1998).
${ }^{79}$ A. Gulans, M. J. Puska, and R. M. Nieminen, Phys. Rev. B 79, 201105 (2009).

${ }^{80}$ J. Klimeš, D. R. Bowler, and A. Michaelides, J. Phys.: Condens. Matter 22, 022201 (2010).

${ }^{81}$ G. Henkelman, B. P. Uberuaga, and H. Jónsson, J. Chem. Phys. 113, 9901 (2000).

${ }^{82}$ G. Henkelman and H. Jónsson, J. Chem. Phys. 113, 9978 (2000).

${ }^{83}$ V. A. Ukraintsev and I. Harrison, Surf. Sci. Lett. 286, L571 (1993).

${ }^{84}$ D. L. Meixner and S. M. George, Surf. Sci. 297, 27 (1993).

${ }^{85}$ Y. Matsumoto, Y. A. Gruzdkov, K. Watanabe, and K. Sawabe, J. Chem. Phys. 105, 4775 (1996).

${ }^{86}$ K. Watanabe and Y. Matsumoto, Surf. Sci. 390, 250 (1997).

${ }^{87}$ A. F. Carlsson and R. J. Madix, Surf. Sci. 458, 91 (2000).

${ }^{88}$ E. Dombrowski, E. Peterson, D. Del Sesto, and A. L. Utz, Catal. Today 244, 10 (2015).

${ }^{89}$ H. Guo and B. Jackson, J. Phys. Chem. C 119, 14769 (2015).

${ }^{90}$ F. Viñes, Y. Lykhach, T. Staudt, M. P. A. Lorenz, C. Papp, H. P. Steinrück, J. Libuda, K. M. Neyman, and A. Görling, Chem. - Eur. J. 16, 6530 (2010).

${ }^{91}$ K. Lee, E. D. Murray, L. Kong, B. I. Lundqvist, and D. C. Langreth, Phys. Rev. B 82, 081101 (2010).

${ }^{92}$ G. Schoofs, C. Arumainayagam, M. Master, and R. Madix, Surf. Sci. 215, 1 (1989).

${ }^{93}$ H. B. Lyon and G. A. Somorjai, J. Chem. Phys. 44, 3707 (1966).

${ }^{94}$ W. H. Weinberg, J. Chem. Phys. 57, 5463 (1972).

${ }^{95}$ W. Takeuchi and Y. Yamamura, Surf. Sci. 277, 351 (1992).

${ }^{96}$ F. Nattino, A. Genova, M. Guijt, A. S. Muzas, C. Diaz, D. J. Auerbach, and G. J. Kroes, J. Chem. Phys. 141, 124705 (2014).

${ }^{97}$ M. J. Murphy and A. Hodgson, J. Chem. Phys. 108, 4199 (1998).

${ }^{98}$ A. C. Luntz and J. Harris, Surf. Sci. 258, 397 (1991).

${ }^{99}$ C. H. Greene and R. N. Zare, Annu. Rev. Phys. Chem. 33, 119 (1982).

${ }^{100}$ W. R. Simpson, T. P. Rakitzis, S. A. Kandel, A. J. Orr-Ewing, and R. N. Zare, J. Chem. Phys. 103, 7313 (1995).

${ }^{101}$ Z. Xie, J. M. Bowman, and X. Zhang, J. Chem. Phys. 125, 133120 (2006).

${ }^{102}$ D. M. Brink and G. R. Satchler, Angular Momentum (Oxford University Press, 1968).

${ }^{103}$ H. Hou, S. J. Gulding, C. T. Rettner, A. M. Wodtke, and D. J. Auerbach, Science 277, 80 (1997).

${ }^{104}$ L. Chen, H. Ueta, R. Bisson, and R. D. Beck, Rev. Sci. Instrum. 84, 053902 (2013).

${ }^{105}$ H. Ueta, L. Chen, R. D. Beck, I. Colon-Diaz, and B. Jackson, Phys. Chem. Chem. Phys. 15, 20526 (2013).

${ }^{106}$ M. P. Schmid, P. Maroni, R. D. Beck, and T. R. Rizzo, Rev. Sci. Instrum. 74, 4110 (2003).

${ }^{107}$ D. A. King and M. G. Wells, Proc. R. Soc. A 339, 245 (1974).

${ }^{108}$ P. M. Hundt, R. Bisson, and R. D. Beck, J. Chem. Phys. 137, 074701 (2012). 